\title{
Star Formation Histories of the LEGUS Dwarf Galaxies. I. Recent History of NGC 1705, NGC 4449, and Holmberg II $^{*}$
}

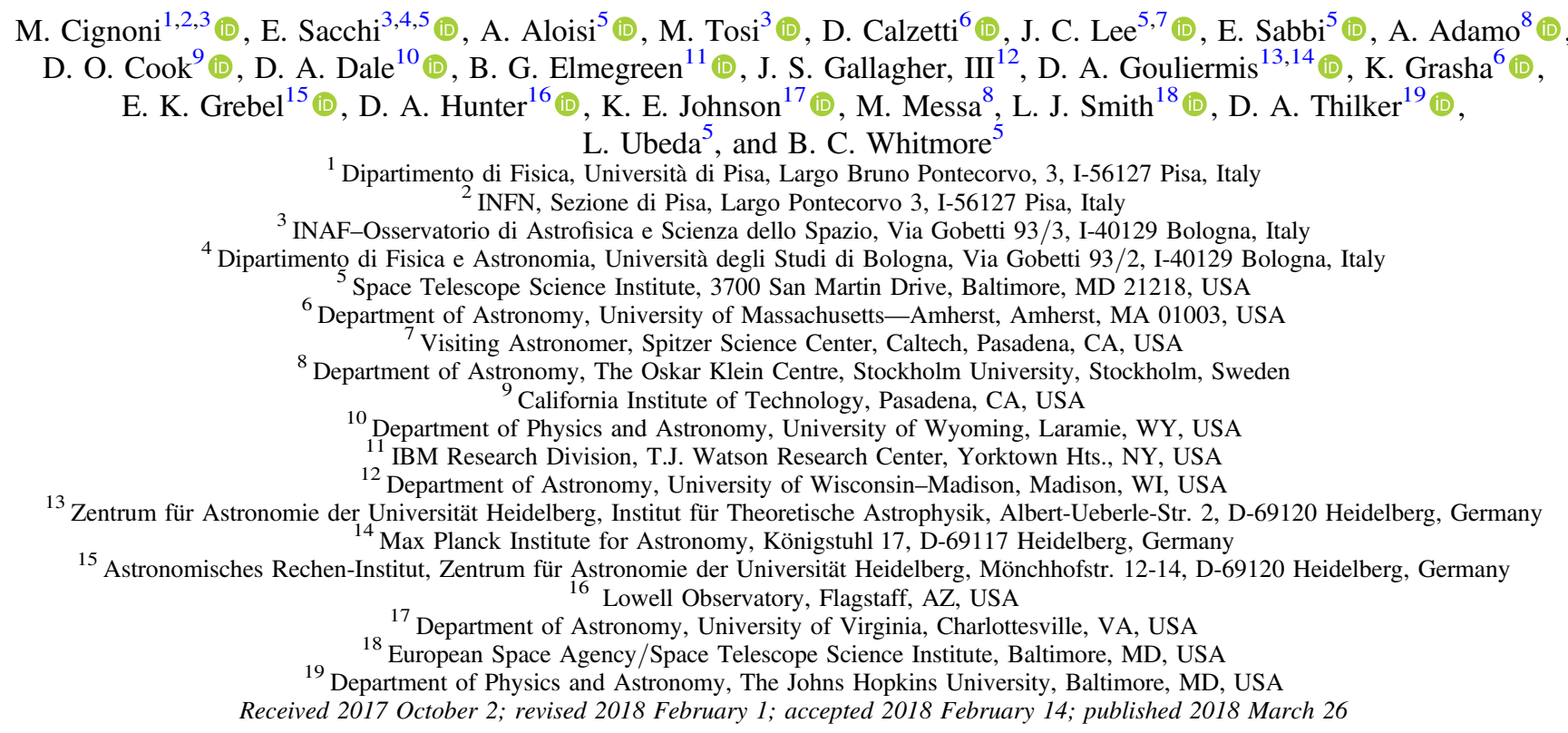

\begin{abstract}
We use Hubble Space Telescope observations from the Legacy Extragalactic UV Survey to reconstruct the recent star formation histories (SFHs) of three actively star-forming dwarf galaxies, NGC 4449, Holmberg II, and NGC 1705, from their UV color-magnitude diagrams (CMDs). We apply a CMD fitting technique using two independent sets of stellar isochrones, PARSEC-COLIBRI and MIST, to assess the uncertainties related to stellar evolution modeling. Irrespective of the adopted stellar models, all three dwarfs are found to have had almost constant star formation rates (SFRs) in the last 100-200 Myr, with modest enhancements (a factor of 2) above the $100 \mathrm{Myr}$ averaged SFR. Significant differences among the three dwarfs are found in terms of the overall SFR, the timing of the most recent peak, and the SFR/area. The initial mass function of NGC 1705 and Holmberg II is consistent with a Salpeter slope down to $\approx 5 M_{\odot}$, whereas it is slightly flatter, $s=-2.0$, in NGC 4449. The SFHs derived with the two different sets of stellar models are consistent with each other, except for some quantitative details, attributable to their input assumptions. They also share the drawback that all synthetic diagrams predict a clear separation in color between the upper main-sequence and helium-burning stars, which is not apparent in the data. Since neither differential reddening, which is significant in NGC 4449, nor unresolved binaries appear to be sufficient to fill the gap, we suggest this calls for a revision of both sets of stellar evolutionary tracks.
\end{abstract}

Key words: galaxies: dwarf - galaxies: formation - galaxies: starburst - galaxies: stellar content Hertzsprung-Russell and $\mathrm{C}-\mathrm{M}$ diagrams - stars: massive

\section{Introduction}

Dwarf galaxies are important. Given the ubiquity of lowmetallicity galaxies at high redshift, understanding how star formation (SF) proceeds in such regimes is crucial for cosmological purposes. Dwarf galaxies lack spiral density waves and differential rotation, hence, global dynamics presumably plays a minor role in regulating their SF. Moreover, while massive galaxies have formed most of their stars in the first $\sim 3 \mathrm{Gyr}$, low-mass galaxies have been forming stars over the entirety of cosmic time. This results in a variety of star formation histories (SFHs; see, e.g., Tolstoy et al. 2009;

\footnotetext{
* Based on observations obtained with the NASA/ESA Hubble Space Telescope at the Space Telescope Science Institute, which is operated by the Association of Universities for Research in Astronomy under NASA Contract NAS 5-26555.
}

McQuinn et al. 2011; Weisz et al. 2011; Gallart et al. 2015) and specific star formation rates (sSFR), ranging from nearly inactive, such as the transition-type dwarf Leo $\mathrm{T}$, to extremely active, such as the blue compact dwarfs (BCDs).

Potential mechanisms for triggering SF in dwarfs can be broadly categorized into either internally driven or externally driven events. From the internal point of view, intrinsic processes such as stochastic self-propagating SF (Gerola et al. 1980) certainly play a role, in particular at the low-mass end where single star-forming events can change the galaxy luminosity significantly. However, BCDs appear to be different from non-starbursting dwarf irregulars in terms of their $\mathrm{H} \mathrm{I}$ and internal dynamics. The higher central rotation-velocity gradients of BCDs suggest a relation between the intense activity and the gravitational potential (Lelli et al. 2012). Moreover, 
despite their isolation, BCDs exhibit star-forming regions that are kinematically decoupled from the rest of the galaxy (Koleva et al. 2014). Elmegreen et al. (2012) suggested that giant starforming clumps in dwarf irregular galaxies could inspiral toward the center, feeding intense SF. This is because in such small galaxies, star-forming clumps could be sufficiently massive to exceed a few percent of the galaxy mass enclosed inside their orbital radii, therefore producing dynamically significant torques on dark matter halo particles, halo stars, and the surrounding disk to lose their angular momentum on a timescale of 1 Gyr. Other proposed internal mechanisms are the effect of massive triaxial dark halos, promoting the migration of a significant fraction of gas from the periphery to the center of the dwarf (Bekki \& Freeman 2002), or bars made of dark matter (Hunter \& Elmegreen 2004).

External processes must also be important. If cold dark matter (CDM) is scale-free as predicted, groups of dwarf galaxies are a natural expectation on small mass scales, and dwarf galaxies could be formed through the same physical process as massive galaxies. While dwarfs accreting dwarfs have already been observed in at least one case, DDO 68 (Annibali et al. 2016; Sacchi et al. 2016), dwarf groups have only been recently observed by Stierwalt et al. (2017) using the panchromatic TiNy Titans (TNT; Stierwalt et al. 2015) survey, a systematic study of SF in interacting dwarf galaxies. According to this study, the interaction between dwarf galaxies could be quantitatively different compared to more massive counterparts. In fact, both paired dwarfs and paired massive galaxies show enhanced SF out to separations of $\sim 100 \mathrm{kpc}$ (e.g., Patton et al. 2013; Stierwalt et al. 2015), but the effect in dwarfs is stronger by a factor of 1.3 and involves a larger fraction of the virial radius. Dwarfs are therefore more globally affected by small companions. A large fraction of interacting dwarfs are starbursting, too. If one defines as starbursts those regions with a global $\mathrm{H} \alpha$ equivalent width $>100 \AA$, Stierwalt et al. (2017) found that starbursts occur in $20 \%$ of the TNT isolated pairs, compared to only $6 \%$ of the matched isolated single dwarfs, suggesting that dwarf-dwarf interactions play a key role in triggering starbursts in dwarfs. Interestingly, the final coalescence stage of the dwarf-dwarf merger is not required to produce starbursts, but rather they are triggered at earlier stages of the interaction. Finally, collisions between dwarfs and satellites that are predominantly star-free, or even dark, could explain the strong bursts of SF that occur in some dwarfs without apparent cause (Starkenburg et al. 2016). Indeed, H I clouds with no evident optical counterpart have been found around several BCDs (Ramya et al. 2009), suggesting that infall of such gas clouds is not an unlikely event.

In this framework, quantitative information on the SFH in dwarf galaxies (see e.g., Weisz et al. 2011) is fundamental in order to understand the connection between the observed activity and processes such as merging, accretion, and interaction. The SFH likely keeps a record of the past interactions. Detailed high-resolution SFH studies may also provide invaluable hints on how star-forming regions evolve and what turns off SF, from the smallest to galactic-size scales, and how these processes depend on dynamical effects.

The purpose of this paper is to quantify the most recent SFH of individual galaxies using the UV color-magnitude diagrams (CMDs) of their resolved stars. While all studies of SFH performed so far (e.g., Tolstoy et al. 2009 and references therein; Weisz et al. 2011) aimed to cover the longest possible lookback time and infer the SFH back to the earliest epochs, in this case we aim to resolve in higher detail the most recent epochs. Here we exploit the Legacy ExtraGalactic UV Survey (LEGUS; Calzetti et al. 2015) to study at high temporal resolution the most recent past of three actively star-forming dwarf galaxies, NGC 1705, NGC 4449, and Holmberg II (hereafter Ho II, also known as UGC 4305). All three dwarfs have had their optical SFH already derived by others (see e.g., Annibali et al. 2003; Weisz et al. 2008; McQuinn et al. 2010), but our study is complementary to theirs since it deals only with the last 100-200 Myr but with the higher temporal resolution achieved with UV photometry. Moreover, we derive the SFHs by adopting two different and independent sets of isochrones to estimate the uncertainties resulting from different approaches and the input physics (rotation, mixing length, amount of overshooting, etc.) of the stellar models. We also take advantage of the higher capability of separating mainsequence (MS) and post-MS stars in UV CMDs to evaluate how well the evolutionary models are able to reproduce the observed properties of massive stars, an important test for stellar theories.

Table 1 describes the relevant properties of these galaxies that could be gathered from the literature. The three galaxies show different degrees of isolation, with NGC 1705 being the most isolated and NGC 4449 the least isolated. The most massive one, NGC 4449, is similar in size and mass to the Large Magellanic Cloud (see, e.g., Karczewski et al. 2013). This galaxy has been classified as a "global" starburst, since SF appears to involve much of the galaxy (Hunter 1997). Its closest neighbor is at a projected distance of $41 \mathrm{kpc}$, and previous studies (Martínez-Delgado et al. 2012; Rich et al. 2012) have reported evidences of interactions and of a stellar tidal stream. The second most massive system, Ho II, is similar to the Small Magellanic Cloud in terms of absolute magnitude, H I, and total mass (Puche et al. 1992). VLA H I observations (Puche et al. 1992) have shown a large number of H I shells and holes in the ISM, probably caused by stellar feedback from multiple generations of SF spread out over tens or hundreds of Myr (Weisz et al. 2009). The closest neighbor of Ho II, Kar 52, is at a projected distance of $30 \mathrm{kpc}$. The least massive dwarf, NGC 1705, is also the most gas poor and isolated (its closest neighbors, LSBG F157-089 and MRSS 157-121650, are at more than $500 \mathrm{kpc}$; see, e.g., Firth et al. 2006, Evstigneeva et al. 2007). This galaxy is the only one hosting a super star cluster (SSC; $\left.M_{B}=-14.5\right)$ and exhibiting strong galactic winds (see, e.g., Meurer et al. 1992). The isolation makes NGC 1705 an ideal candidate to understand how long the SF can be sustained in isolation and whether the activity is linked to the accretion of cold gas from the intergalactic medium. Indeed, cold flows of gas are predicted by $\Lambda$ CDM models of galaxy formation (Kereš et al. 2005). NGC 1705 is also the only one morphologically classified as a BCD, while the other two are "irregulars."

The SFHs are recovered using the synthetic CMD approach applied to the F336W versus F336W - F555W CMD. This filter combination is ideal for identifying the youngest populations in our galaxies, mostly traced by MS, Hertzsprung gap (HG), and core He-burning $(\mathrm{HeB})$ stars more massive than $5 M_{\odot}$. Although we could have used the bluest LEGUS filter available, the $\mathrm{F} 275 \mathrm{~W}$, improving the age resolution in the last $50 \mathrm{Myr}$, this would have exacerbated the effect of reddening, canceling the 
Table 1

Fundamental Properties of NGC 4449, Ho II, and NGC 1705

\begin{tabular}{|c|c|c|c|c|c|c|c|c|}
\hline Galaxy & $\begin{array}{l}\text { R.A. } \\
\text { (J2000) }\end{array}$ & $\begin{array}{l}\text { Decl. } \\
\text { (J2000) }\end{array}$ & $\begin{array}{c}D \\
(\mathrm{Mpc})\end{array}$ & $E(B-V)$ & $\begin{array}{c}M_{\mathrm{H} \mathrm{I}} \\
\left(10^{7} M_{\odot}\right)\end{array}$ & $\begin{array}{c}M_{\star} \\
\left(10^{7} M_{\odot}\right)\end{array}$ & $12+\log (\mathrm{O} / \mathrm{H})$ & $M_{B}$ \\
\hline NGC 4449 & 124442.7 & -355800 & $3.82 \pm 0.18(a)$ & 0.20 (a) & $300 \pm 77(\mathrm{~g})$ & 110(i) & $8.26(d)$ & $-18.2(\mathrm{j})$ \\
\hline Ho II & 124600.4 & -335017 & $3.38(b)$ & 0.03 (b) & 73(h) & 23(i) & 7.92(f) & $-16.7(\mathrm{k})$ \\
\hline
\end{tabular}

Note. (a) Annibali et al. (2008), (b) Dalcanton et al. (2012), (c) Tosi et al. (2001), (d) Berg et al. (2012), (e) Moustakas et al. (2010), (f) Croxall et al. (2009), (g) Lelli et al. (2014), (h) Huchtmeier \& Richter (1989), (i) Calzetti et al. (2015) and references therein, (j) Hunter et al. (1999), (k) Tully (1988), (1) Annibali et al. (2015).

resolution gain. Moreover, it would have shortened the lookback time reachable by the photometry.

This paper is organized as follows. In Section 2, we describe observations and data reduction. In Section 3, we discuss the spatial distribution, and we compare the observed CMDs with stellar evolution models. In Section 4, we describe the SFH recovery technique. In Section 5 , we present the recovered SFHs. Finally, Sections 6 and 7 summarize our results.

\section{Observations}

The observations are part of the LEGUS survey, a Hubble Space Telescope (HST) panchromatic stellar survey of 50 nearby star-forming dwarf and spiral galaxies. The goal of the survey is to investigate scales and modes of SF by leveraging the UV imaging. The scientific objectives and the data reduction are described in Calzetti et al. (2015), while the stellar photometry is described in detail in Sabbi et al. (2018). The observations were performed with the Wide Field Camera 3 (WFC3) and Advanced Camera for Surveys (ACS) in parallel, in a set of broad bands over the range $0.27-0.81 \mu \mathrm{m}$, respectively, F275W, F336W, F438W, F555W, and F814W (equivalent to $N U V, U, B, V$, and $I$, respectively) and combined with archival optical ACS data.

Resolved stellar photometry was performed using the DOLPHOT package (e.g., Dolphin 2000). DOLPHOT performs point-spread function fitting on all the flat-fielded and CTE-corrected images per field simultaneously. We then used the main DOLPHOT routine to make photometric measurements in each filter independently on the preprocessed images, relative to the coordinate system of the drizzled reference image. The output photometry from DOLPHOT is on the calibrated VEGAMAG scale (see Sabbi et al. 2018).

\section{Data \\ 3.1. Spatial Distributions}

Figure 1 shows the spatial distributions of the resolved stars in the three galaxies (from top to bottom: NGC 4449, Ho II, and NGC 1705); the left column shows the F336W images, the middle column shows the map in physical units (pc) of all stellar sources identified both in F336W and in F555W, colorcoded by the density estimated using a Gaussian kernel (standard deviation $0.2 \mathrm{pc}$ ), and the right column shows zoomins of the densest subregions ( $\approx 600 \mathrm{pc}$ wide). In order to make the plots easily comparable, the color maps are normalized to the same limits.

The three galaxies have very different morphologies. NGC 1705 is characterized by a single roundish overdensity, whereas NGC 4449 and Ho II have multiple overdensities that are irregularly distributed. Zooming in on the densest subregions (right panels) reveals other differences. NGC 4449's peak subregion is very crowded (as indicated by the almost uniform red color), with multiple peaks (black areas) of higher density. Compared to NGC 4449, Ho II's subregion has a much lower average density (most of the area is green), with only three peaks at the same density of NGC 4449. NGC 1705's subregion has intermediate properties between NGC 4449 and Ho II, except in the very center, where it is even denser than NGC 4449 (the very center is extremely crowded, as evidenced by the "ring" shape of the black region, ${ }^{20}$ a sign that we are missing stars in the center). In conclusion, on average, NGC 4449, NGC 1705, and Ho II represent a sequence of decreasing average density. On the other hand, when only the peak density is considered, the sequence from the highest to the lowest becomes NGC 1705, NGC 4449, and Ho II.

\section{2. $C M D s$}

In order to reject non-stellar objects and to have a clean final sample of stars for our CMDs, we applied quality cuts. The DOLPHOT output was filtered to only allow objects classified as stars with signal-to-noise ratio $>5$ in both filters. In addition, we selected detections for which DOLPHOT reports an error flag of 3 or less. The list was further culled using sharpness $(<0.15)$ and crowding $(<1.3)$. Our final star catalogs for NGC 4449, Ho II, and NGC 1705 contain 107,761, 11,516 and 3741 stars, respectively. The resulting "cleaned" CMDs are shown in Figure 2 together with the latest PARSEC-COLIBRI (PAdova and TRieste Stellar Evolution Code version 1.2S plus COLIBRI code for asymptotic giant branch (AGB) thermal pulses; Bressan et al. 2012; Tang et al. 2014; Marigo et al. 2017) isochrones for the ages 3, 10, 20, 50, 100, and $200 \mathrm{Myr}$ (corrected for reddening $E(B-V)$ and distance moduli 0.1 and 27.9, for NGC 4449, 0.03 and 27.6 for Ho II, and 0.045 and 28.5 for NGC 1705). The adopted metallicity is $Z=0.005$ for NGC 4449 and NGC 1705 , and 0.002 for Ho II.

Guided by the isochrones, we recognize three common stellar phases in the CMDs of Figure 2: a prominent blue plume of MS stars, and an $\mathrm{HG}$ and a $\mathrm{HeB}$ phases. Typical masses populating these CMDs are larger than $5 M_{\odot}$ and as massive as $80 M_{\odot}$. Given the depth of these CMDs, the maximum age that can be investigated in NGC 4449, Ho II, and NGC 1705 is about $180 \mathrm{Myr}, 200 \mathrm{Myr}$, and $100 \mathrm{Myr}$, respectively.

Besides the similarities, these CMDs also show some marked differences in morphology that cannot be fully explained by observational effects. Most notably, the MS of NGC 4449 appears broader than that of either NGC 1705 or Ho II. Moreover, the luminosity distribution of $\mathrm{HG} / \mathrm{HeB}$ stars appears (1) rather uniform in NGC 4449, (2) to decline with increasing luminosity in Ho II, and (3) clumpy in NGC 1705.

\footnotetext{
${ }^{20}$ This is certainly related also to the presence of the SSC at the center of NGC 1705.
} 

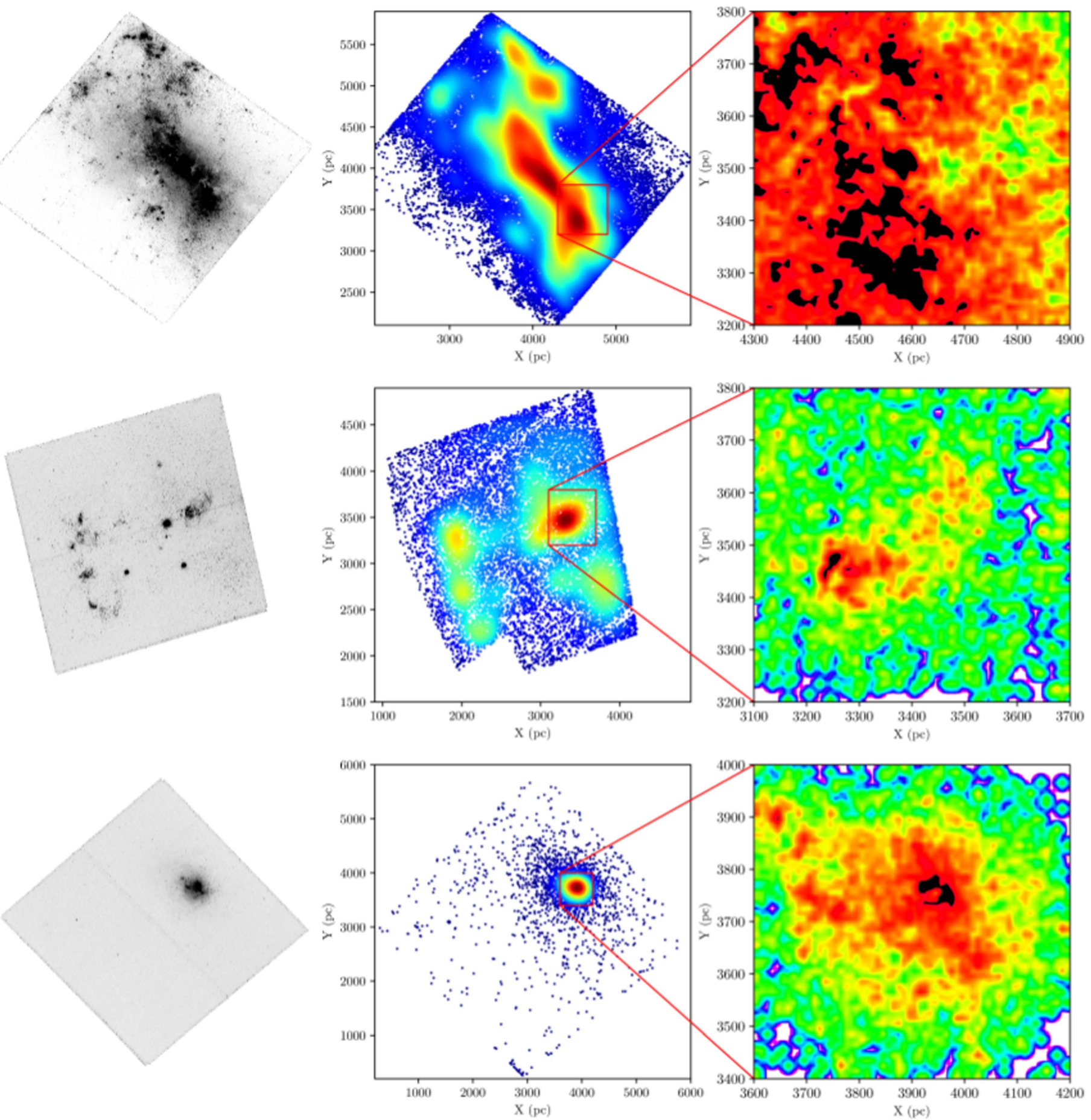

Figure 1. Left column: F336W images of NGC 4449 (top panel), Ho II (middle panel), and NGC 1705 (bottom panel). Middle column: all stellar sources detected in F336W and F555W, color-coded by density (estimated with a Gaussian kernel). Right column: zoom-in on the densest subregions.

Concerning the MS spread, it seems unlikely that a metallicity variation can explain it all, despite the higher metallicity of NGC 4449. In fact, in massive hot stars, the opacity is dominated by electron scattering, which depends only on the mass fraction of hydrogen $X$ as $0.20(1+X)$ and is about constant at $Z<0.01$. Moreover, the observed MS in Ho II and NGC 1705 is bracketed by the $3 \mathrm{Myr}$ isochrone, which encompasses the blue edge, and the MS termination point (MSTP; the massive analogue of the MS turnoff) of all isochrones, defined by the red extent of MS evolution (visible as a hook in all isochrones older than $3 \mathrm{Myr}$ ). This feature suggests that age, rather than photometric errors (modest), unresolved binaries, or differential reddening, is the dominant driver of the MS width in Ho II and NGC 1705.

On the other hand, the MS of NGC 4449 is significantly broader $^{21}$ than expected from age spread alone. Given the youth of the population, it appears very probable that

\footnotetext{
${ }^{21}$ The MS width is measured with the standard deviation of the color distribution of stars in the magnitude interval $22<\mathrm{F} 336 \mathrm{~W}<23$.
} 


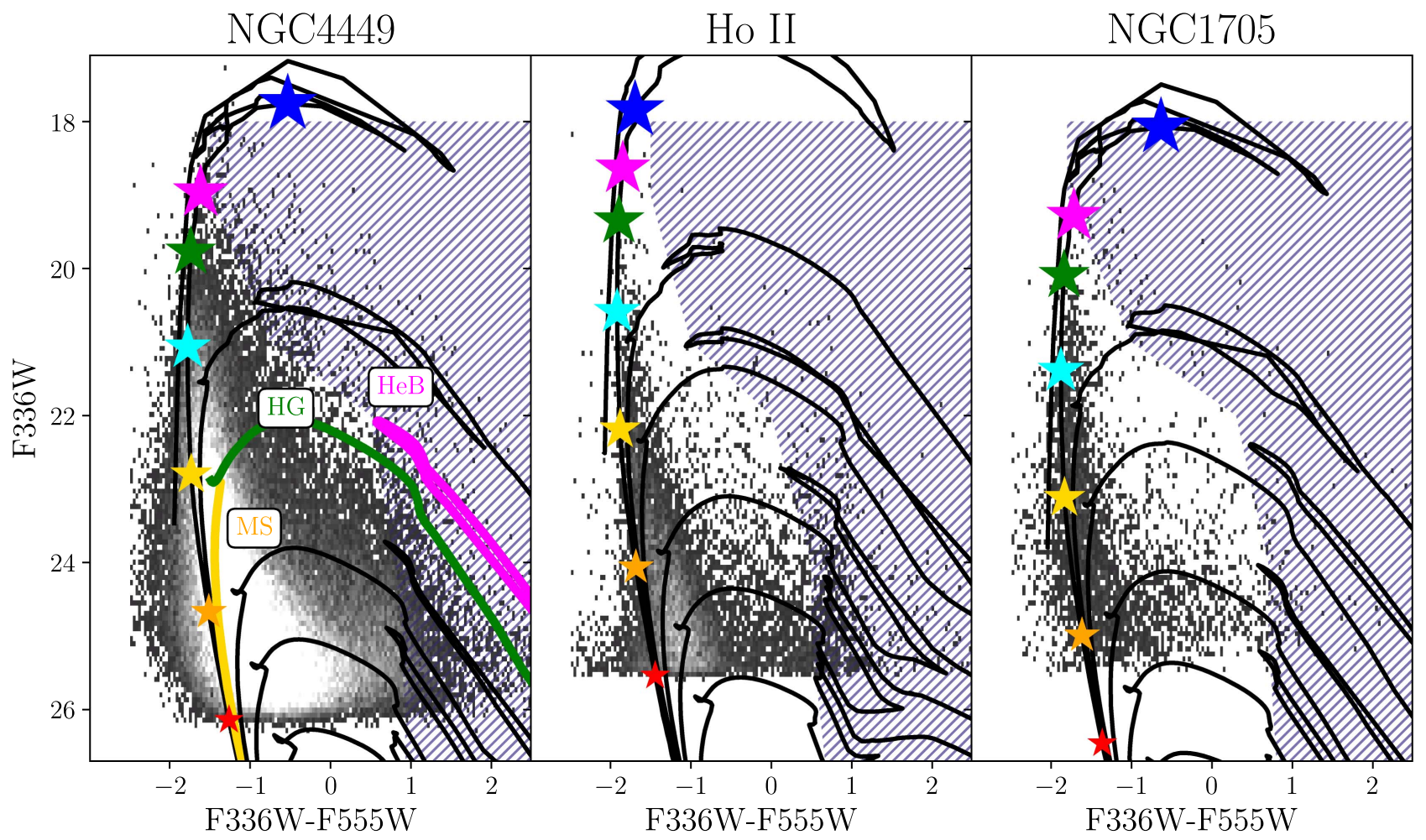

Figure 2. From left to right: CMDs for NGC 4449, Ho II, and NGC 1705. PARSEC-COLIBRI isochrones of ages 3, 10, 20, 50, 100, and 200 Myr are overlaid. The most relevant stellar phases are also indicated along the $20 \mathrm{Myr}$ isochrone (leftmost panel) with lines of different colors: orange for MS, green for HG, and magenta for $\mathrm{HeB}$. The adopted distance modulus, foreground reddening $E(B-V)$, and metallicity $Z$ of each galaxy are, respectively, 27.9, 0.1, and 0.005 (for NGC 4449); 27.6, 0.03, and 0.002 (for Ho II); and 28.5, 0.045, and 0.005 (for NGC 1705). The hatched area indicates the region of the CMD populated by HeB stars. Star symbols represent PARSEC-COLIBRI stellar models of $3 \mathrm{Myr}$ and masses of 6 (red), 10 (orange), 20 (gold), 40 (cyan), 60 (green), 70 (magenta), 80 (blue) $M_{\odot}$.

differential reddening is artificially inflating the apparent width of the MS. Indeed, using far-ultraviolet (FUV) and Balmer-line imagery for 22 OB complexes in NGC 4449, Hill et al. (1994) derived reddening values $E(B-V)$ ranging from 0.25 to 0.6 from the Balmer decrement. Alternatively, stellar rotation (see, e.g., Meynet \& Maeder 1997) or larger core-convective overshooting (see, e.g., Bressan et al. 2015) could be also responsible for the broadening of the MS. However, such effects should occur also in Ho II and NGC 1705, whereas only in NGC 4449 is the MS broader than expected from age spread.

For intermediate/massive stars beyond the MS, the stellar phase before the red giant branch (RGB) is so fast (KelvinHelmholtz timescale) that the probability of observing stars is low compared to the nuclear phases. Generally, this causes the so-called HG, i.e., the observed lack (or paucity) of stars in the evolutionary phase right after the MS. However, stellar production in these galaxies is such that the number of stars in these fast evolutionary phases is not negligible. Indeed, HG stars are copious in NGC 4449, with no sign of interruption between $\mathrm{F} 336 \mathrm{~W} \approx 19$ and the faint end of the CMD. In contrast, the bulk of HG stars in Ho II is fainter than F336W $\approx 21$, while the distribution of HG stars in NGC 1705 seems bimodal, with overdensities around $\mathrm{F} 336 \mathrm{~W} \approx 21$ (part of these objects could be $\mathrm{HeB}$ stars) and below $\mathrm{F} 336 \mathrm{~W} \approx 22$.

Concerning the $\mathrm{HeB}$ phase, theory predicts that the bluest point in the blueward excursion of the core $\mathrm{HeB}$ phase for masses above the $2.5 M_{\odot}$ (the so-called "Blue Loop," BL) depends on several quantities, including stellar mass (more massive stars having more extended loops), metallicity (more metal-rich stars having less extended blue loops), and input

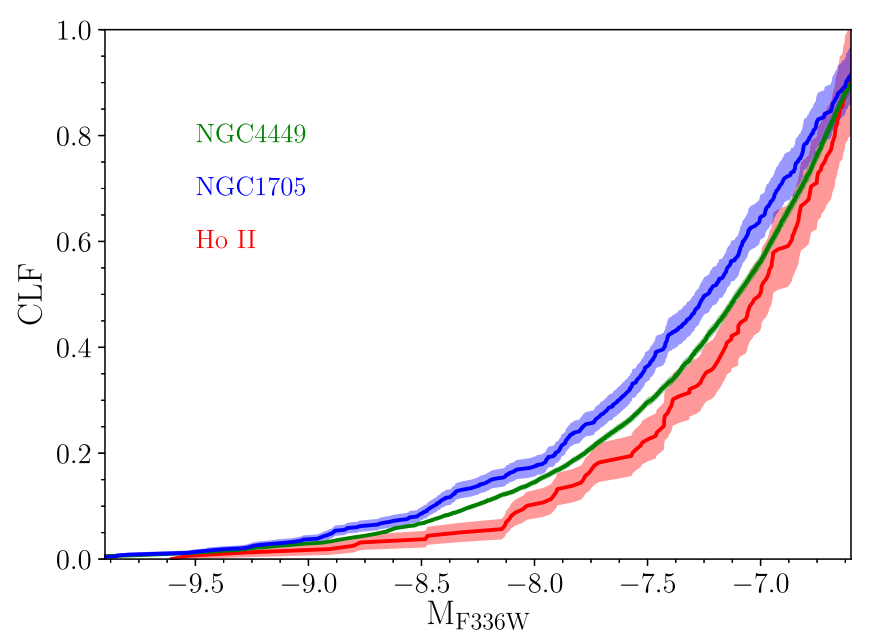

Figure 3. Cumulative luminosity functions, in absolute magnitude, for NGC 4449 (green solid line), Ho II (red solid line), and NGC 1705 (blue solid line). The shaded envelopes represent the Poisson uncertainty $(1 \sigma)$.

physics (see, e.g., Tang et al. 2016). On the other hand, the luminosity of the BL is mainly driven by the stellar mass. In fact, the energy budget during the HeB phase is provided by the $\mathrm{HeB}$ core and the H-burning shell. Since above $2.5 M_{\odot}$ the He core increases with increasing total mass as a consequence of the increasing mass of the convective core during the previous core H-burning phase, the HeB luminosity depends on the mass of the star, providing a useful mass-luminosity relation (and, in turn, a luminosity-age relation). 

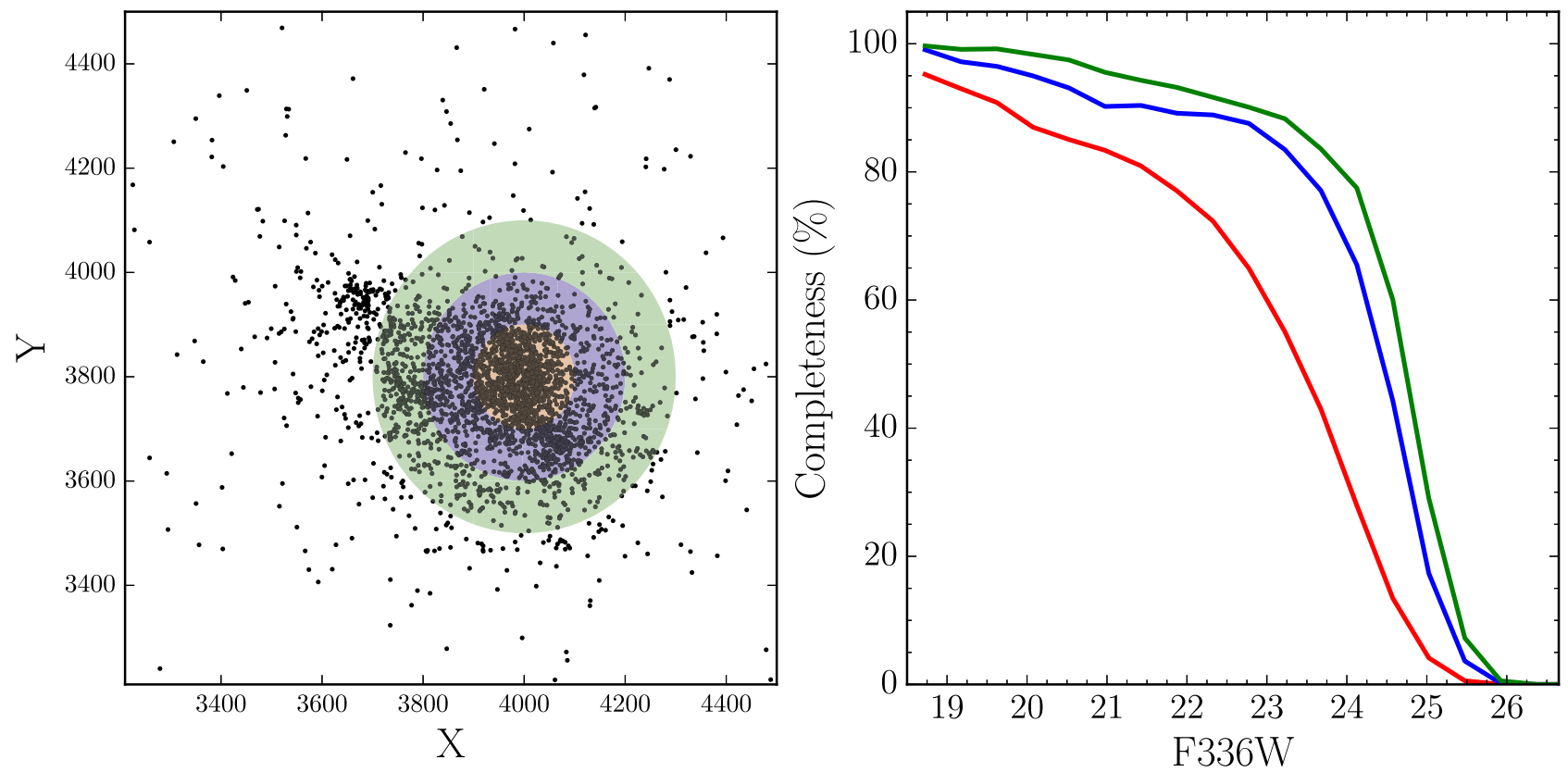

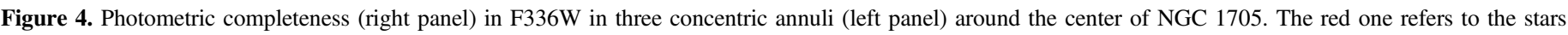

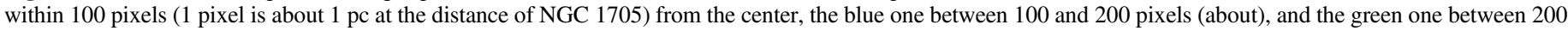
and 300 pixels.

The comparison with the isochrones in Figure 2 suggests that in all three galaxies the blue edge of the BL is redder than $\mathrm{F} 336 \mathrm{~W}-$ $\mathrm{F} 555 \mathrm{~W} \approx 0.5$ for stars fainter than 22 (see the hatched area) and reaches $\mathrm{F} 336 \mathrm{~W}-\mathrm{F} 555 \mathrm{~W} \approx-1.5$ at magnitudes around 19. Clearly, the relatively high metallicity of these galaxies $(Z=0.002-0.005)$ prevents the $\mathrm{HeB}$ phase from reaching the MS color. The net result is that, depending on the magnitude, stars at intermediate colors are a different mixture of $\mathrm{HG}$ and BL stars. In Ho II and NGC 1705, the CMD region located between $18<\mathrm{F} 336 \mathrm{~W}<21$ and $-0.5<\mathrm{F} 336 \mathrm{~W}-\mathrm{F} 555 \mathrm{~W}<0$ is likely populated by both $\mathrm{HG}$ and BL stars, whereas the CMD region between $21<\mathrm{F} 336 \mathrm{~W}<25$ and $\mathrm{F} 336 \mathrm{~W}-\mathrm{F} 555 \mathrm{~W} \approx 0$ is mostly populated by HG stars. The situation is more ambiguous in NGC 4449, where the color spread tends to smear out many CMD features already at magnitudes as bright as 24 .

Since HG and BL monotonically fade as the population age increases, both stellar species are fundamental clocks to infer the recent SFH of a galaxy. The route of the BL as an age indicator has been pioneered by Dohm-Palmer et al. (1997) in the dwarf irregular galaxy Sextans A and recently applied to several other dwarf irregulars by, e.g., McQuinn et al. (2011, 2012).

The main advantage of $\mathrm{HG} / \mathrm{BL}$ over the $\mathrm{MS}$ is that subsequent generations of $\mathrm{HG} / \mathrm{BL}$ stars do not overlap each other as they do on the MS. Moreover, the $\mathrm{HG} / \mathrm{BL}$ is on average brighter than the coeval MSTP. However, since the MS evolutionary times are much longer than the post-MS times, star counts along the MS are statistically more robust. This property becomes crucial in galaxies like NGC 1705 and Ho II, whose low global SFR does not provide a sufficient number of post-MS stars. In the next sections, we will use the HG and BL stars in synergy with MS stars to infer the recent $\mathrm{SFH}$ of the three dwarfs.

Before closing this section, we demonstrate that the comparison of the luminosity function (LF) for the three dwarfs provides a measure of the relative SFRs. To do this, since the total number of stars in NGC 4449 is significantly higher than in the other two, we rely on the cumulative LF
(CLF). Figure 3 shows the CLFs for the three galaxies corrected for the corresponding distance modulus and reddening $E(B-V)$ (see Table 1), normalized to the number of stars with absolute magnitude $M_{\mathrm{F} 336 \mathrm{~W}}$ brighter than $-6.5^{22}$ (corresponding to stars younger than $\approx 15 \mathrm{Myr}$ ). The CLF of NGC 1705 increases faster than that of the other two, which are similar to each other (compatible within $1 \sigma$ as shown by the error bars). Since the number of stars brighter than a certain magnitude is proportional to the average SFR younger than a certain age, the CLF is also a measure of the relative SFR (normalized to the rate $15 \mathrm{Myr}$ ago). In this light, Figure 3 suggests that NGC 1705 is relatively younger than Ho II and NGC 4449, despite the latter being globally more massive. Indeed, above $M_{\mathrm{F} 336 \mathrm{~W}}=-6.5$, NGC 4449 hosts five times more stars than NGC 1705, and thirty times more stars than Ho II. We suggest that the recent activity in these galaxies is not driven by the total mass but rather by external factors or stochastic phenomena.

\section{Synthetic CMDs}

\subsection{Artificial Star Tests}

A mandatory ingredient for meaningful data-model comparisons is to quantify photometric errors and the incompleteness of the data. To this aim, a typical approach is a proper artificial star test, i.e., the injection of "fake" sources (one at a time) onto the actual images: the source detection routine used for our science images is applied to the fields containing the combined science images and the fake sources. Counting how many fake stars are lost as a function of magnitude and position provides the map of the local incompleteness.

Since young stars $(<100-200)$ Myr tend to be concentrated in associations and filaments, most of them will be affected by more significant errors and incompleteness than the average in

\footnotetext{
22 This limit corresponds to an approximate apparent magnitude $\mathrm{F} 336 \mathrm{~W}=21.5$, where star counts are complete in all three galaxies.
} 


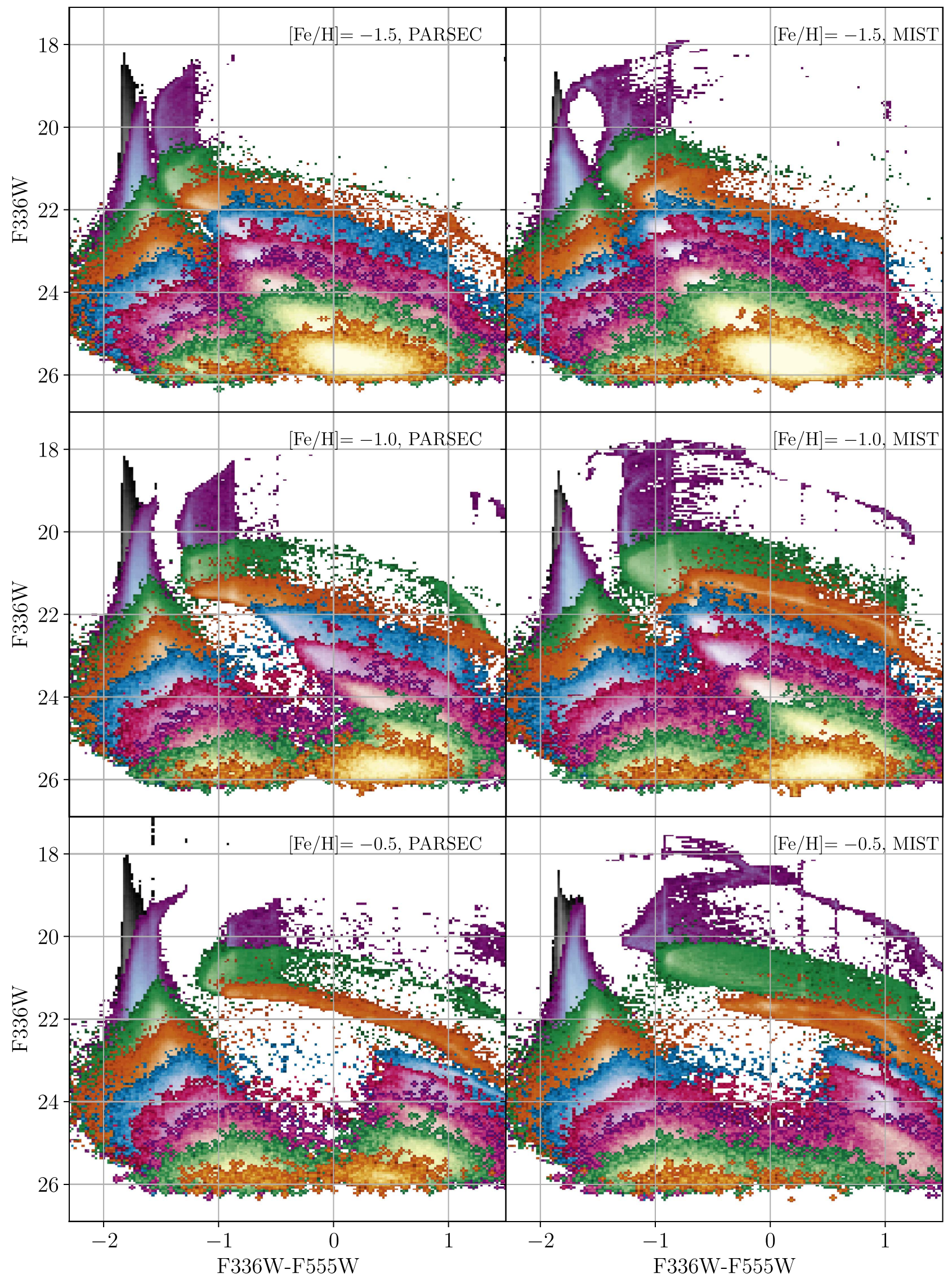

Figure 5. From top to bottom, example of basic synthetic CMDs generated for $[\mathrm{M} / \mathrm{H}]=-1.5,-1.0$, and -0.5 ; errors/incompleteness of NGC 4449 ; binary fraction of 30\%; and distance modulus of NGC 4449 (see Table 1). For each metallicity, different colors represent different star formation episodes with logarithmic duration $\log ($ age $)=5.0-6.6,6.6-7.0,7.0-7.2,7.2-7.4,7.4-7.6,7.6-7.8,7.8-8.0,8.00-8.25$, and 8.25-8.50. The adopted distance and foreground extinction are 27.9 and $E(B-V)=0.1$, respectively. The PARSEC-COLIBRI models are on the left, and the MIST models are on the right. 


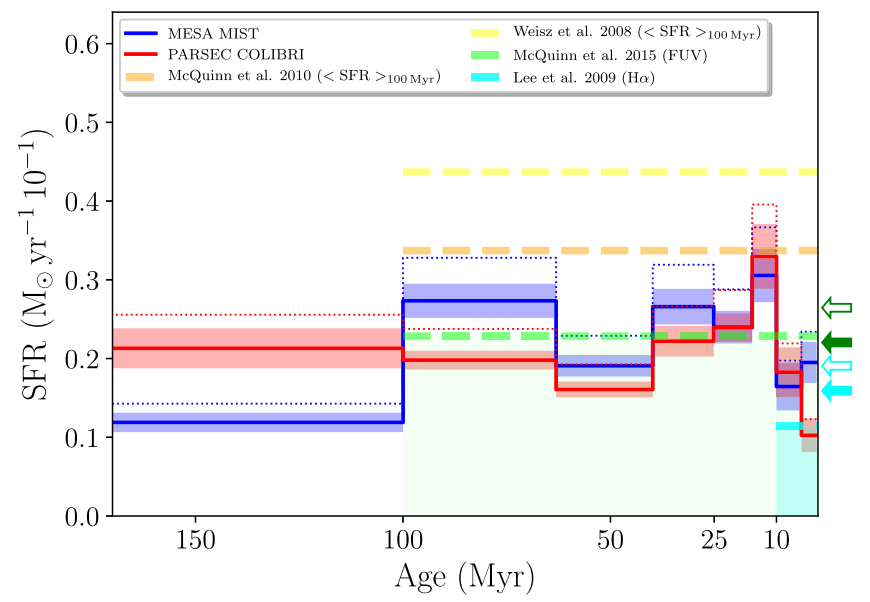

Figure 6. Recovered SFH of Ho II. Blue and red continuous lines represent the MIST and PARSEC-COLIBRI solutions, respectively. The adopted IMF is Kroupa-2001. Thin dotted lines are the corresponding solutions obtained by extrapolating the IMF exponent -2.3 down to $0.1 M_{\odot}$. The green and cyan filled arrows on the right side of the plot stand for our $100 \mathrm{Myr}$ averaged SFR and $10 \mathrm{Myr}$ averaged SFR (averaged between the two solutions), respectively (open arrows are extrapolations using an exponent of -2.3 down to $0.1 M_{\odot}$ ). The orange and yellow dashed lines show the 100 Myr averaged SFR found by, respectively, Weisz et al. (2008) and McQuinn et al. (2010). The green dashed line is the average rate computed by McQuinn et al. (2015) using FUV emission, while the cyan dashed line indicates the average rate from $\mathrm{H} \alpha$ observations computed using the $\mathrm{SFR}(\mathrm{FUV}) / \mathrm{SFR}(\mathrm{H} \alpha)$ conversion factor computed by Lee et al. (2009).

the field. To overcome this problem, we followed the procedure described by Cignoni et al. (2016). As a first step, fake stars are evenly distributed over the galaxy field of view (FoV). The observed density of stellar sources (plagued by incompleteness) is then corrected for the local incompleteness, restoring the "true" profile of the galaxy. As a second step, fake stars are injected following the reconstructed profile. The resulting incompleteness, being weighted with the real stars, will be an unbiased estimate of the actual incompleteness suffered by the young stars.

As an example, Figure 4 shows the estimated F336W completeness profiles (right panel) of NGC 1705 in three concentric regions (see left panel) centered on the densest region of the galaxy. As expected, where crowding is most severe, completeness drops faster. In the central region (red line) the $\mathrm{F} 336 \mathrm{~W}$ catalog is $50 \%$ incomplete at 23.5 , while in more external annuli a $50 \%$ incompleteness is reached at 24.5 (blue line) and 24.75 (green line).

\subsection{SFH Recovery Algorithm}

The SFH of the three galaxies was recovered using the population synthesis code SFERA (Star Formation Evolution Recovery Algorithm). The analysis details are described in Cignoni et al. (2015, 2016). Here, we provide only a short description of the procedure; a complete discussion is available in the papers mentioned above.

A library of "basic" synthetic CMDs is generated using the isochrones from the adopted sets of stellar models. To test the systematic uncertainties due to the stellar models, in our procedure the synthetic CMDs are generated by adopting either the PARSEC-COLIBRI or the MIST (MESA Isochrones and Stellar Tracks) isochrones (Paxton et al. 2011, 2013, 2015; Choi et al. 2016; Dotter 2016). The two sets differ both in the input physics and in the assumptions about the efficiency of macroscopic mechanisms, like rotation (PARSEC-COLIBRI models are static, MIST models are rotating with $v / v_{\text {crit }}=0.4$ ). Each basic synthetic CMD is a Monte Carlo realization with a constant SFH (in a given bin of ages) and fixed metallicity $( \pm 0.05 \mathrm{dex})$. In this work we adopted nine logarithmic time bins, namely $\log ($ age $)=5.0-6.6,6.6-7.0,7.0-7.2,7.2-7.4$, 7.4-7.6, 7.6-7.8, 7.8-8.0, 8.00-8.25, and 8.25-8.50. Concerning metallicity, we allowed the code to use metallicities ${ }^{23}$ between $[\mathrm{M} / \mathrm{H}]=-2$ and 0.1 with a resolution of $0.1 \mathrm{dex}$. Once the metallicity and ages are selected, each basic CMD is populated with a Kroupa (2001) initial mass function (IMF) between 0.1 and $300 M_{\odot}$. Other IMF slopes are considered if no SFH is found to match the data. The last step concerns the binary population: $30 \%$ of synthetic stars are considered to be part of unresolved binary systems, and their flux is combined with a companion, whose mass is a random fraction between 0 and 1 of the primary.

Concerning distance and extinction, we adopted the following approaches. Given the relatively low sensitivity of the UV SFH to the assumed distance (mainly due to the rapid evolutionary phases populating the UV CMD), we opted to use literature distances (see Table 1) based on the tip of the RGB (see, e.g., Bellazzini et al. 2001), a robust feature missing in the UV CMDs but clearly visible in optical CMDs. On the other hand, the high sensitivity of the UV color to the reddening distribution allowed us to model reddening and SFH simultaneously. The extinction distribution is modeled with two parameters: a foreground extinction applied evenly to all stars, $\mathrm{AV}$, and a differential extinction, $d \mathrm{AV},{ }^{24}$ that is applied in addition to AV. Moreover, we adopted the extinction law of Cardelli et al. (1989) and assumed a normal total-to-selective extinction value of $\mathrm{Rv}=3.1$.

To mimic the observational effects and uncertainties, each synthetic CMD is then convolved with photometric errors (derived from the cumulative distribution of the mag output $_{-}$ mag $_{\text {input }}$ of fake stars) and incompleteness derived from the artificial star tests.

Finally, the combination of basic CMDs that minimizes the residuals with respect to the observational CMD (in terms of Poissonian likelihood) is searched with the hybrid-genetic algorithm SFERA. Figure 5 shows an example of basic synthetic CMDs (PARSEC-COLIBRI on the left, MIST on the right) for three different metallicities $([\mathrm{M} / \mathrm{H}]=-0.5,-1.0$, $-1.5)$. At fixed metallicity, the differences between the PARSEC-COLIBRI and MIST synthetic CMDs are indicative of the different underlying stellar physics and stellar evolution codes. When deriving the SFH in the next section, these differences will serve as an indication of the potential differences between a given model and the "truth."

Figure 5 also confirms the low dependence on metallicity in this range of ages. The only significant effect concerns the color extension of the BL, which shrinks with increasing metallicity. Given this difficulty of establishing the metallicity, we opted to use the available spectroscopic information (see Table 1) together with the photometry to provide additional constraints on the SFH. In particular, (1) the metallicity in the last 10 Myr was fixed at the spectroscopic value of the observed $\mathrm{H}$ II regions (within the measured errors), and (2) we limited the

\footnotetext{
23 To be consistent with their own assumptions, we adopt the approximation $[\mathrm{M} / \mathrm{H}]=\log \left(Z / Z_{\odot}\right)$, with $Z_{\odot}=0.0152$ for the PARSEC-COLIBRI models and $Z_{\odot}=0.0142$ for the MIST models.

${ }^{24}$ Each star is assigned a random additional extinction between 0 and $d \mathrm{AV}$.
} 


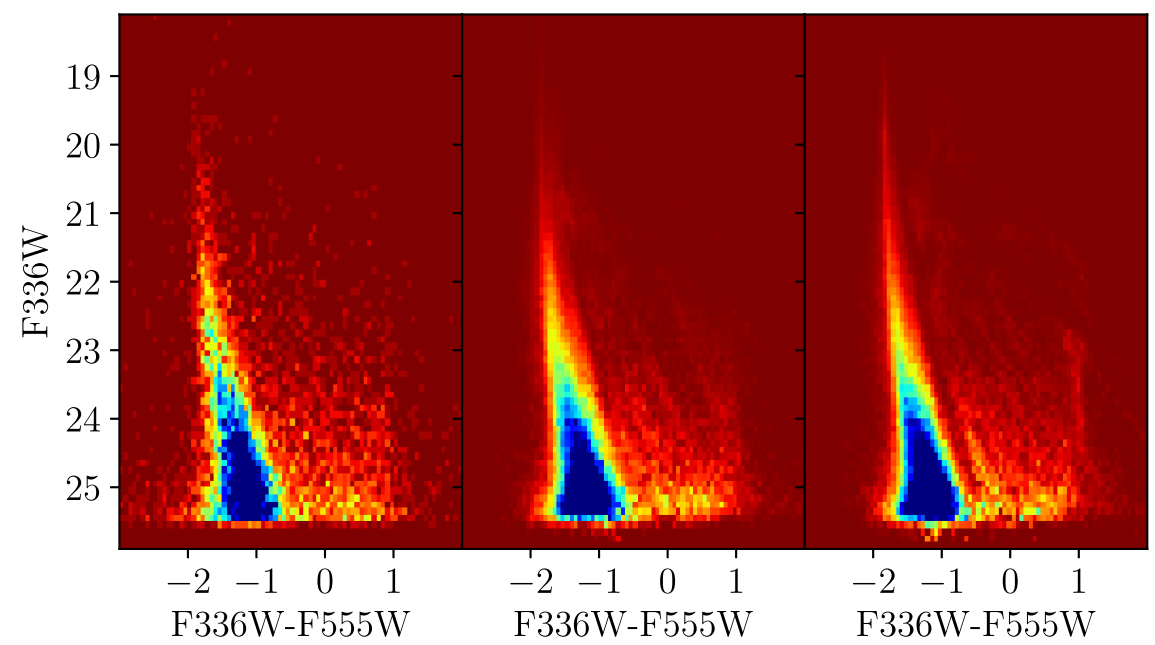

Figure 7. Ho II's CMD (left panel) compared to the best synthetic CMDs generated with the PARSEC-COLIBRI (middle panel) and MIST (right panel) isochrones.

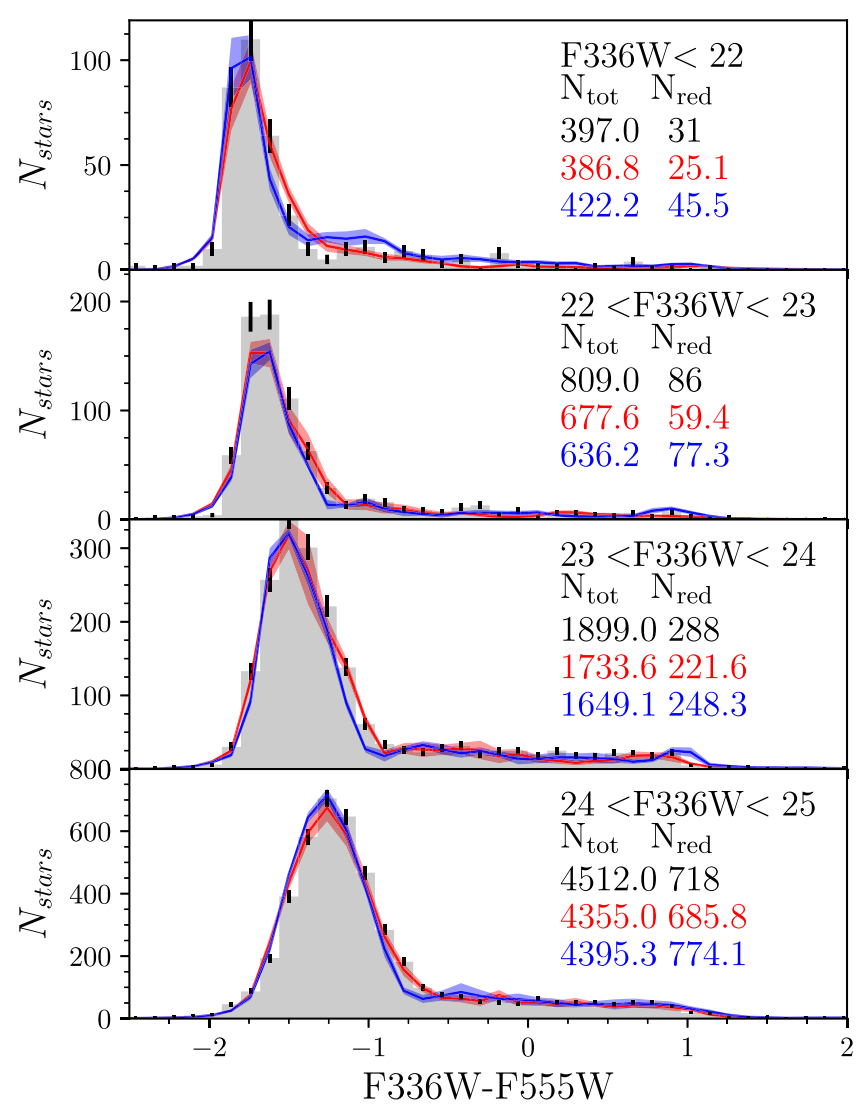

Figure 8. Color distribution of Ho II (gray shaded area), the best PARSECCOLIBRI model (red line), and the best MIST model (blue line) for the labeled magnitude ranges. The observed and predicted counts (total and redder than color -0.5 ) are also shown.

number of free parameters by imposing that the metallicity be an increasing function of time.

Throughout this work, we adopt the oxygen abundance as a tracer of the overall recent metallicity (in the last $10 \mathrm{Myr}$ ).

\section{SFHs}

\subsection{Ho II}

Figure 6 shows the recovered SFHs for Ho II. Red and blue continuous lines are for the PARSEC-COLIBRI and MIST models, respectively, while the dotted lines are the same SFHs extrapolated with the IMF exponent $s=-2.3$ down to $0.1 M_{\odot}$. The green and cyan filled arrows on the right side of the plot line indicate our $100 \mathrm{Myr}$ averaged SFR and $10 \mathrm{Myr}$ averaged SFR (averaged between the two solutions), respectively (open arrows are extrapolations using an exponent of -2.3 down to $0.1 M_{\odot}$ ). Orange, yellow, green and cyan thick dashed lines are instead SFRs taken from the literature (see below). Overall, the results obtained using the two sets of isochrones are qualitatively similar. This is not surprising because the CMD of Ho II is dominated by MS stars, for which different evolutionary models generally agree. Both solutions are consistent with an almost constant SF activity over the last $180 \mathrm{Myr}$, with mild enhancements whose intensity is at most 1.5 higher than the $100 \mathrm{Myr}$ averaged SFR $\left(0.022 \mathrm{M}_{\odot} \mathrm{yr}^{-1}\right.$; see the green filled arrow on the right side of the plot). The SFH obtained with the MIST models is less constant, with a broad enhancement between 60 and $100 \mathrm{Myr}$ ago. The SFH with the PARSECCOLIBRI models shows a similar recent peak, although the general activity is more constant. The best foreground extinction AV is $0.25 \mathrm{mag}$, while the differential one, $d \mathrm{AV}$, is $0.02 \mathrm{mag}$.

Dalcanton et al. (2012) found similar recent SF enhancements using HST/ACS optical CMDs. They derived the relative SFH (normalized to the lifetime averaged rate) for two different regions dubbed UGC4305-1 and UGC4305-2. The latter is the one with a larger overlap with our UV observations, so we used its SFH for comparison with our solution. The main difference between the two is the timing of the most recent peak, which is younger than $10 \mathrm{Myr}$ in their solution whereas it is between 10 and $16 \mathrm{Myr}$ ago in ours. The ratio between the peak rate and the average activity in the last $200 \mathrm{Myr}$ is instead very similar, with Dalcanton et al.'s (2012) ratio only slightly higher (2 versus 1.5 ).

Weisz et al. (2008) derived the optical SFH for the entire area covered by the HST/ACS observations (UGC4305-1 plus UGC4305-2) and found that Ho II has been about two times more active in the last $20 \mathrm{Myr}$ than in the last $200 \mathrm{Myr}$. A similar behavior is also found in McQuinn et al. (2010) using the same data and approach. The yellow and orange thick dashed lines in Figure 6 show that their 100 Myr averaged SFRs decreased by a factor of $2.25,{ }^{25}$ which is the ratio between the number of bright stars $(\mathrm{F} 555 \mathrm{~W}<24)$ found in the two ACS pointings and in our WFC3 pointing (we also note that both Weisz et al. 2008 and

\footnotetext{
$\overline{25}$ This number was estimated using the optical CMDs (Sabbi et al. 2018) for the two $H S T /$ ACS pointings.
} 


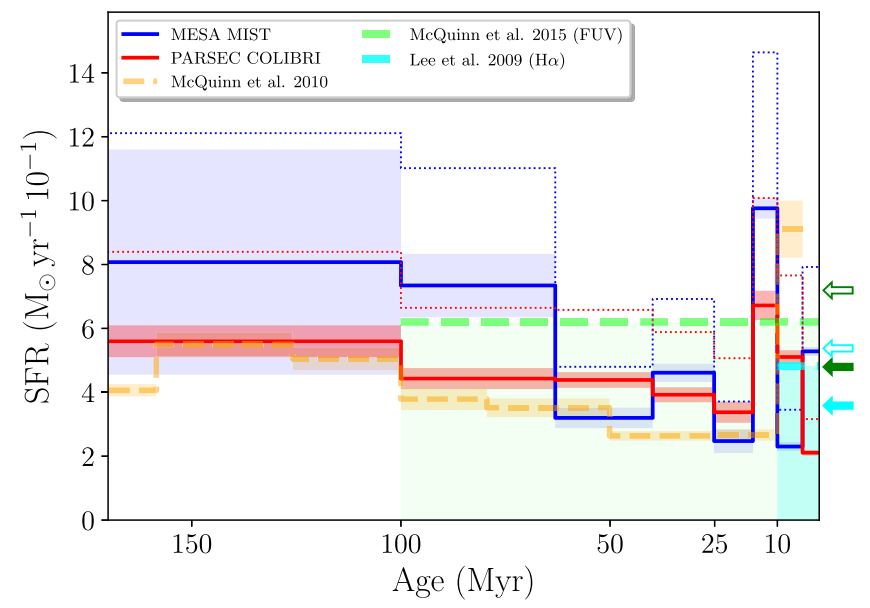

Figure 9. Recovered SFH of NGC 4449. The adopted IMF is a double power law with exponent $s=-2.0$ above $0.5 M_{\odot}$ and $s=-1.3$ below. The symbols are the same as in Figure 6.

McQuinn et al. 2010 used a Salpeter IMF down to $0.1 M_{\odot}$ ). After this correction, these rates are only slightly higher than our average rate extrapolated with an IMF exponent of -2.3 down to $0.1 M_{\odot}$ (open arrows on the right side of Figure 6). Moreover, the effect of using different stellar models (our models are based on the most updated evolutionary tracks) and filters (our F336W filter combined with the F555W filter provides a better temperature resolution for blue stars compared to F555W - F814W) could explain the slightly older age of our most recent peak.

Another useful comparison is with current SFR indicators like $\mathrm{H} \alpha$ nebular emission and FUV non-ionizing continuum emission. While the first indicator arises from the recombination of gas ionized by the most massive $\mathrm{O}$ - and early-type B-stars, therefore tracing the SF in the last few million years, the UV flux stems from the photospheres of O- through latertype B-stars, and thus traces the SF in the last $100 \mathrm{Myr}$. In Figure 6, the average SFRs derived from FUV and $\mathrm{H} \alpha$ emission are shown by the thick green and cyan dashed lines, respectively (both rates assume a Salpeter IMF down to $\left.0.1 M_{\odot}\right)$. The former combines the SFR-FUV calibration from Kennicutt (1998) and the GALEX FUV flux, cropped to the footprint of the HST/ACS observations (taken from McQuinn et al. 2015) and corrected for the factor 2.25. The latter uses the ratio $\mathrm{SFR}(\mathrm{H} \alpha) / \mathrm{SFR}(\mathrm{FUV})$ provided by Lee et al. (2009). Overall, both the SFR(FUV) and $\operatorname{SFR}(\mathrm{H} \alpha)$ are in excellent agreement with our findings.

The most basic check on the reliability of the recovered $\mathrm{SFH}$ is to compare the synthetic and observed CMDs. Figure 7 shows the best synthetic CMDs (the middle panel corresponds to the PARSEC-COLIBRI simulation, while the right-hand panel corresponds to the MIST one) corresponding to the recovered SFH compared to the data (left hand panel).

The color distribution of data versus simulations in four bins of magnitude is shown in Figure 8. Both models reproduce the observations very well, with only a few appreciable misfits. The colors and, in particular, the width of the simulated MS match fairly well those of the observed one, in all the magnitude bins. The total number and color distribution of stars redder than F336W $-\mathrm{F} 555 \mathrm{~W}=-0.5$, likely bona fide $\mathrm{HG}$ and BL stars, are also well-matched. For this galaxy, a powerlaw IMF with exponent $s=-2.3$ down to $5 M_{\odot}$ is consistent with the data.
For the post-MS, we notice some mild issues in the color distribution. In the brightest bin of Figure 8 (top panel), the model distribution shows an excess of $\mathrm{HeB}$ stars with colors between -1 and -1.4 (corresponding to the clump of stars at $\mathrm{F} 336 \mathrm{~W}=20-21$ in the synthetic CMDs of Figure 7). Another general difference concerns how the post-MS phase is populated. In the CMDs of Figure 7, it is clear that model stars tend to lie either near or far from the MS, whereas the observed post-MS distribution is rather continuous in color.

It is unclear what triggered the increases in SF, since Ho II is a rather isolated galaxy, at the very edge of the M81 group, almost detached from it. There are no signs of interaction between Ho II and close-by members of the M81 group-Kar 52 and UGC 4483 are located at a projected distance of $\sim 30 \mathrm{kpc}$ and $\sim 105 \mathrm{kpc}$, respectively. From an orbital point of view, taking into account the relative radial velocity between Ho II and Kar 52, an interaction might have happened $600 \mathrm{Myr}$ ago, and so differential rotation should have dissipated the effects of a weak interaction. Moreover, since the dynamical mass of Ho II is a factor of 100 greater than that of Kar 52 (Melisse \& Israel 1994), the latter is unlikely to have significantly affected Ho II.

Using FUV, H $\alpha$, and H I observations, Stewart et al. (2000) studied the possible internal mechanisms triggering SF activity in Ho II. The authors found that the energy deposited into the ISM by supernovae and stellar winds are sufficient to account for the observed properties of the $\mathrm{H}$ I distribution.

From deep VLA data, Bureau \& Carignan (2002) found that the distribution of neutral hydrogen has a comet-like morphology - compressed on one side with a faint extended component on the opposite side - with the tail pointing away from the center of the M81 group. The authors suggested that ram pressure from a hot intragroup medium could be responsible for the $\mathrm{HI}$ morphology, although tidal stripping could not be ruled out.

More recently, using deep, wide-field optical Subaru images, Bernard et al. (2012) studied the stellar populations in the outskirts of Ho II. They found very few, if any, Ho II stars beyond a galactocentric distance of $\sim 7 \operatorname{arcmin}$, where $\mathrm{HI}$ is instead present and becomes distorted. Since tidal forces would affect gas and stars equally, they argued that ram pressure is the likely culprit of the H I morphology. Under this light, the interaction between the ISM and the intergalactic medium (IGM) could be responsible for the recent SFR enhancement. Indeed, some ram pressure models (see, e.g., Bekki \& Couch 2003) indicate that the pressure of the IGM can induce the collapse of molecular clouds and consequently trigger a burst of SF. Bernard et al. (2012) also found that old RGB stars are more centrally concentrated than young blue stars, which follow the (compressed) H I contours.

\section{2. $N G C 4449$}

The SFHs in better agreement with the observational CMD are shown in Figure 9 (the red and blue continuous lines are for the PARSEC-COLIBRI and MIST models, respectively). This galaxy experienced a significant event between 10 and $16 \mathrm{Myr}$ ago, when its activity increased by a factor of $\sim 1.5-2$ above the 100 Myr averaged SFR (indicated with a filled green arrow on the right side of the plot). SF has continued since that peak event at a lower level, consistent with the activity prior the peak. The best extinction value, AV, is $0.18 \mathrm{mag}$, while the differential one, $d \mathrm{AV}$, is $1.09 \mathrm{mag}$. This solution was obtained using an IMF exponent $s=-2.0$ (instead of the canonical $s=-2.3$ ) above $5 M_{\odot}$, since no combination of SFH and extinction matching the 


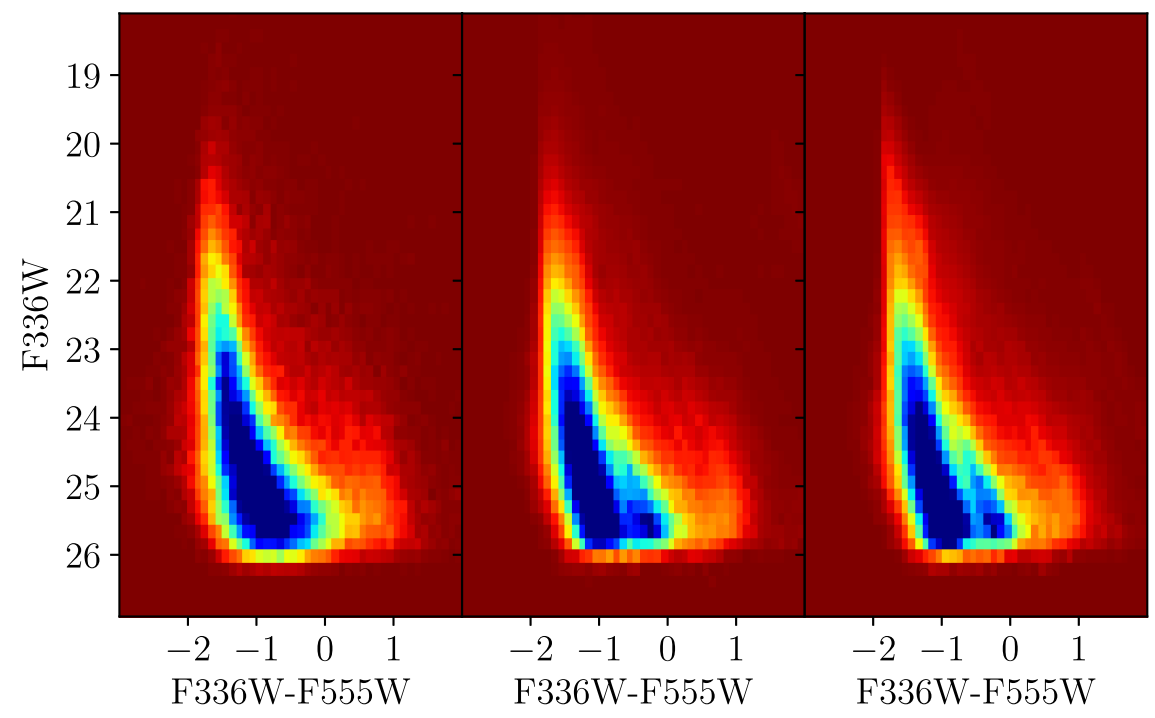

Figure 10. Observational CMD of NGC 4449 (left panel) compared to the best synthetic CMDs (middle and right panels). The symbols are the same as in Figure 7 .

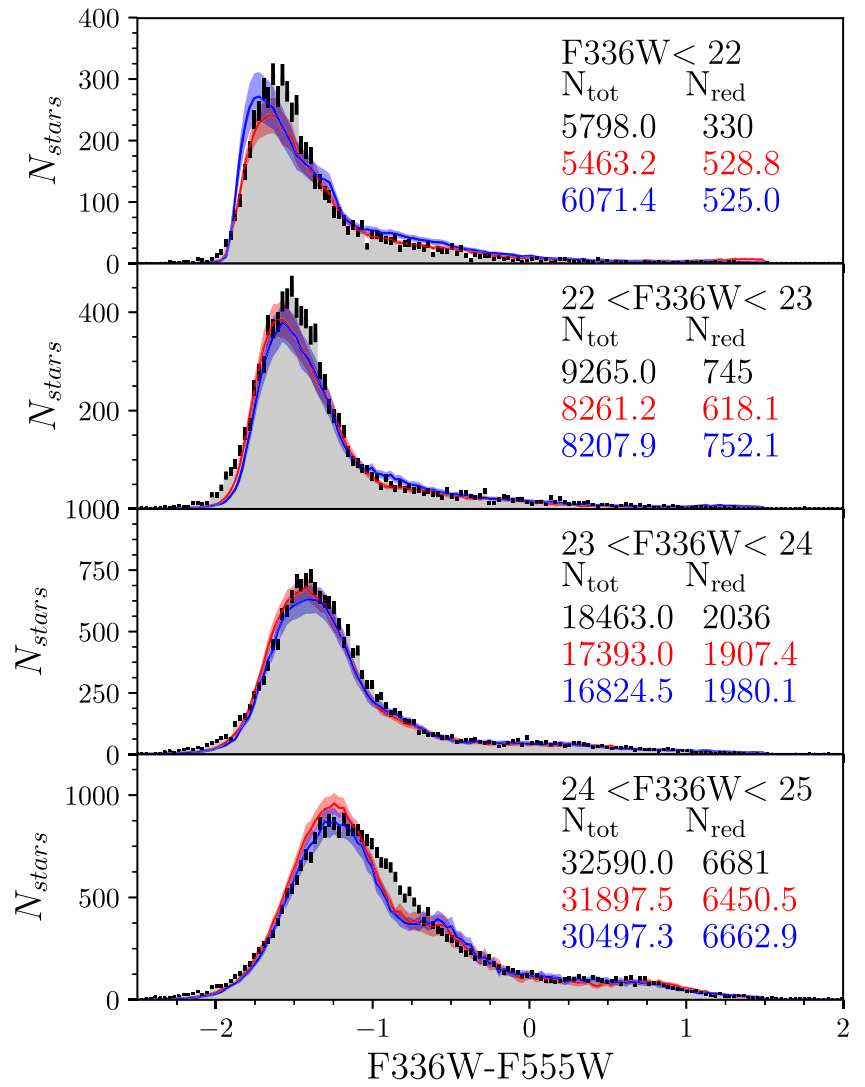

Figure 11. Color distribution of NGC 4449 compared to the models computed with IMF exponent $s=-2.0$ above $5 M_{\odot}$. The symbols are the same as in Figure 8.

number of stars brighter than $\mathrm{F} 336 \mathrm{~W}=23$ was found with $s=-2.3$ : depending on the adopted stellar model, the latter underpredicts counts for magnitudes brighter than $\mathrm{F} 336 \mathrm{~W}=23$ by $15 \%$ (MIST) and 23\% (PARSEC-COLIBRI). Given the lack of constraints below $5 M_{\odot}$, we arbitrarily chose to use $s=-2.0$ down to $0.5 M_{\odot}$, and $s=-1.3$ below.

The corresponding best synthetic CMDs and color distributions are shown in Figures 10 and 11, respectively. For comparison,

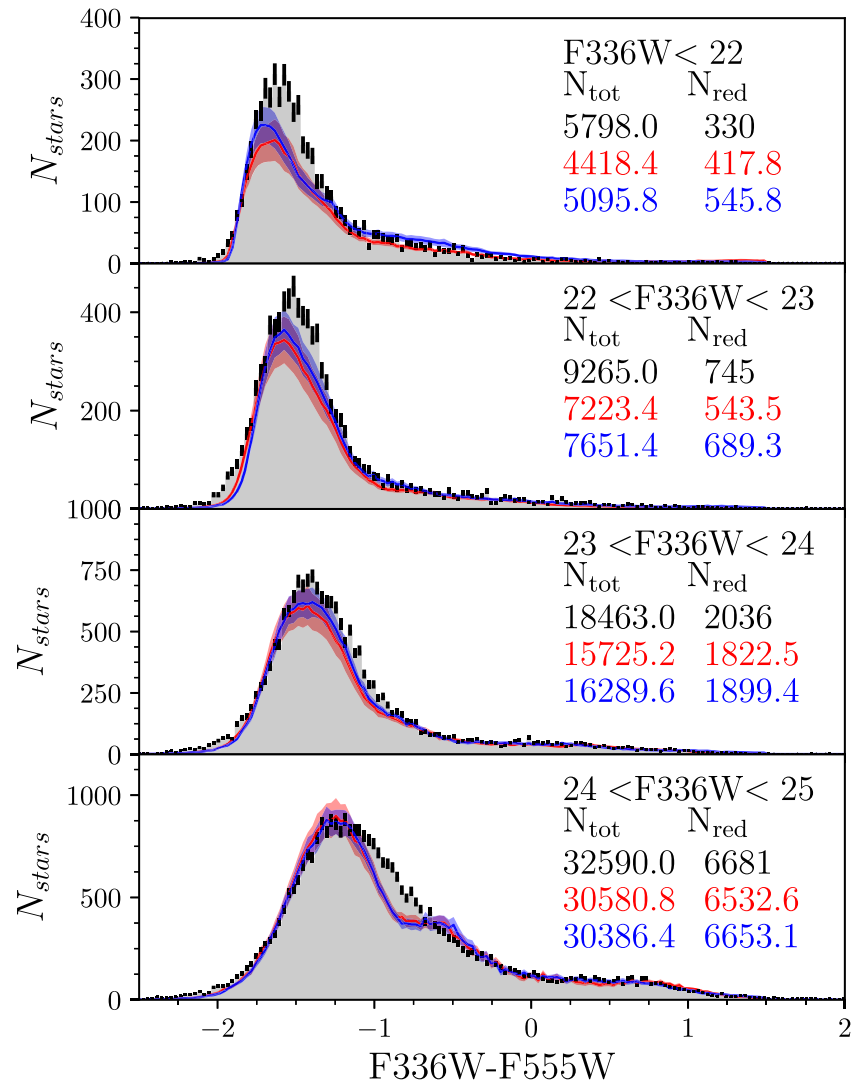

Figure 12. Color distribution of NGC 4449 compared to the models computed with an IMF exponent $s=-2.3$ above $5 M_{\odot}$. The symbols are the same as in Figure 8.

Figure 12 shows the color distribution using the canonical $s=-2.3$. Neither model is perfect. However, models with IMF exponent $s=-2.0$ provide a much better fit to the total number of stars brighter than 23, with minimal differences below this limit. Moreover, irrespective of the adopted IMF, the number of postMS stars (arbitrarily chosen with F336W - F555W $>-0.5$ ) is generally correct, except for $\mathrm{F} 336 \mathrm{~W}<22$ (see the top panel of Figures 11 and 12) where we have an excess of synthetic stars 


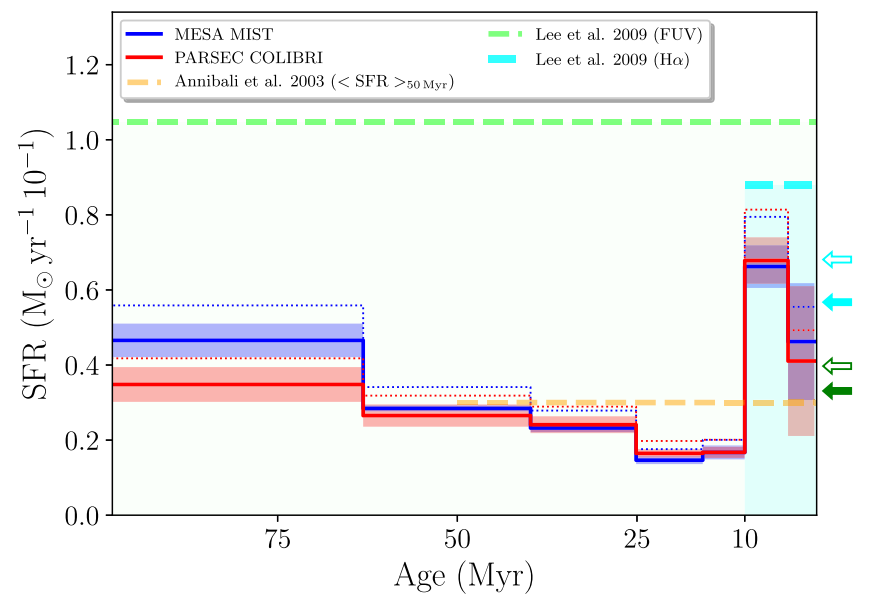

Figure 13. Recovered SFH of NGC 1705. The symbols are the same as in Figure 6.

(slightly exacerbated in the $s=-2.0$ case). Reducing further the IMF exponent has a different effect on the MIST solutions than on the PARSEC-COLIBRI ones. In the former, the general likelihood of the synthetic models worsens, in the latter the likelihood slightly improves. Either way, the excess of synthetic post-MS stars is always exacerbated by a flatter IMF. A better fit could be obtained by varying the mass breakpoint where the IMF flattens, instead of using a single slope above $5 M_{\odot}$. Alternatively, the excess of objects with $\mathrm{F} 336 \mathrm{~W}<23$ could also indicate a high percentage of binaries with similar mass components (as found in starburst clusters; see, e.g., Sana et al. 2013). Following mass accretion and mergers, close binary systems can create a tail of rejuvenated binary products, extending the single star mass function by up to a factor of 2 in mass (Schneider et al. 2015). Finally, we cannot exclude that a fraction of compact, unresolved clusters could be contaminating the upper end of the CMD and LF.

Given these uncertainties and the strong degeneracy between mass and age for very massive stars, we refrain from further discussion of this issue. In terms of SFH, the main effect of adopting a slope of -2 instead of -2.3 is to slightly decrease the recent SFR compared to the old one, hence making the galaxy SF slightly more constant.

At fainter magnitudes $(\mathrm{F} 336 \mathrm{~W}>24$; see the bottom panel of Figures 11 and 12), we have another issue: synthetic models show a drop in the star counts at colors around -1 , whereas the observed color distribution is continuous. This result holds regardless of the adopted IMF. The presence of this dip might be an indication that the models are not able to produce sufficiently extended loops during the $\mathrm{HeB}$ phase. Indeed, a similar dip was already devised by Tang et al. (2014) in several dwarf galaxies. Tang et al. (2014) showed that this discrepancy is overcome by extending the overshooting at the base of the convective envelope. Another possibility is that the differential reddening $d \mathrm{AV}$ is underestimated. In fact, (1) the main effect of differential reddening is to smear CMD features, like the gap between MS and post-MS, and (2) our estimate for $d \mathrm{AV}$ is based on the assumption of flat reddening distribution (each synthetic star is reddened with a random reddening between $\mathrm{AV}$ and $\mathrm{AV}+d \mathrm{AV}$ ), whereas the real distribution might be more complex ${ }^{26}$ (see e.g., Harris et al. 1997) and mass/age dependent. However, the color distribution of MS stars is

\footnotetext{
${ }^{26}$ We also tried a Gaussian reddening distribution with no significant fit improvement.
}

generally broader in the model than in the data, suggesting that $d \mathrm{AV}$ is not significantly overestimated.

The thick orange dashed line in Figure 9 shows the SFH derived by McQuinn et al. (2010) using the same optical ACS images used in this work (Annibali et al. 2008). In order to make a meaningful comparison, McQuinn's et al. SFRs were rescaled to account for the different spatial coverage. In fact, McQuinn's total FoV is more than twice as large as our FoV, being based on two partially overlapping ACS fields, whereas our UV observations consist of one single WFC3 field centered on the central part of the galaxy. The correction factor was estimated by counting the number of young stars inside and outside the WFC3 FoV in our optical CMDs (Sabbi et al. 2018). We found that only $\approx 8 \%$ of the total recent SF in NGC 4449 was missed by our UV observations, hence McQuinn's solution was rescaled down by this amount. Since McQuinn's SFH assumes a Salpeter IMF between 0.1 and $120 M_{\odot}$, we also show our extrapolated rates (thin blue and red dotted lines) using an exponent $s=-2.3$ between $0.1 M_{\odot}$ and $5 M_{\odot}$, and $s=-2.0$ above.

Overall, our rates are within a factor of two of McQuinn's solution. Over the last $180 \mathrm{Myr}$, McQuinn et al. predict a continuous decrease of the SFR interrupted by a very recent and short SF enhancement between 4 (corresponding to the youngest isochrone in their models) and $10 \mathrm{Myr}$ ago. Both our solutions closely follow this behavior, except for the epoch of the recent peak which is slightly older (10-16 Myr ago) in our solution. Part of the difference could be ascribed to the addition of the filter F336W, which is more complete than the optical bands in crowded star-forming regions (in the UV range, most cool stars appear very faint, resulting in less crowding). Other possible causes include (1) the stellar models; those adopted here for intermediate- and high-mass stars are the latest available, whereas those adopted by McQuinn are those from Marigo \& Girardi (2007), which rely on the older Girardi et al. (2000) for masses up to $7 M_{\odot}$, and on Bertelli et al. (1994) above; (2) the different parameterizations of the extinction; and (3) the stellar species used in the fitting process; McQuinn's analysis is based on all stellar species including low-mass stars ascending the RGB and TP-AGBs, while ours is mostly based on high-mass stars along the MS and BL.

Our average rates (filled arrows on the right side of the plot) also compare very well with the $\mathrm{H} \alpha$ and FUV emission rates, as shown in Figure 9 with the thick green and cyan dashed lines, respectively. As already done for Ho II, the FUV SFR adopts the SFR-FUV calibration from Kennicutt (1998) and the GALEX FUV flux, cropped to the footprint of the HST/ ACS observations (taken from McQuinn et al. 2015) and corrected by $8 \%$ to account for the different spatial coverage, while the latter uses the ratio $\operatorname{SFR}(\mathrm{H} \alpha) / \mathrm{SFR}(\mathrm{FUV})$ provided by Lee et al. (2009). Both FUV and $\mathrm{H} \alpha$ rates assume a Salpeter IMF down to $0.1 M_{\odot}$.

An obvious question is what triggered the SF enhancement. If it is a cold flow accretion or a merger event, nearby $\mathrm{H}$ I gas maps might show morphologically disturbed features. Indeed, the global dynamics of the H I associated with NGC 4449 show large distortions. A key observational feature for this galaxy is neutral hydrogen extending to six times its Holmberg radius (see, e.g., van Woerden et al. 1975; Hunter et al. 1998), characterized by an elongated ellipse of lower column density with a major axis of $35 \mathrm{kpc}$. Beyond the ellipse, there is a complex distribution of clouds and streamers that wrap around 


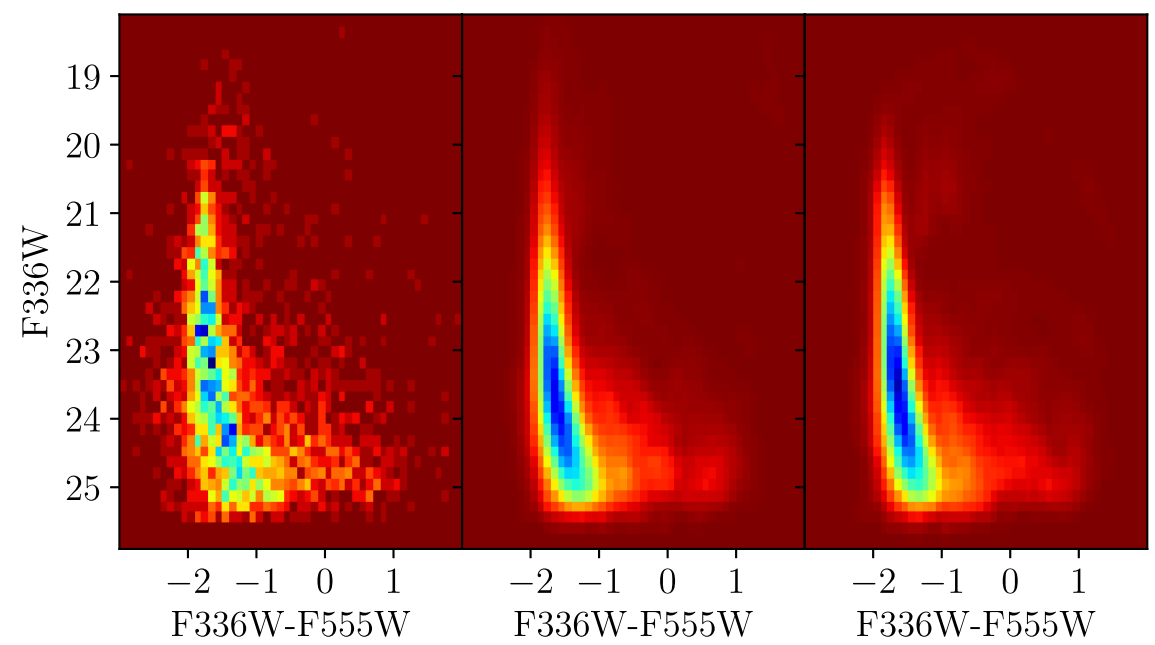

Figure 14. Observational CMD of NGC 1705 (left panel) compared to the best synthetic CMDs (middle and right panels). The symbols are the same as in Figure 7.

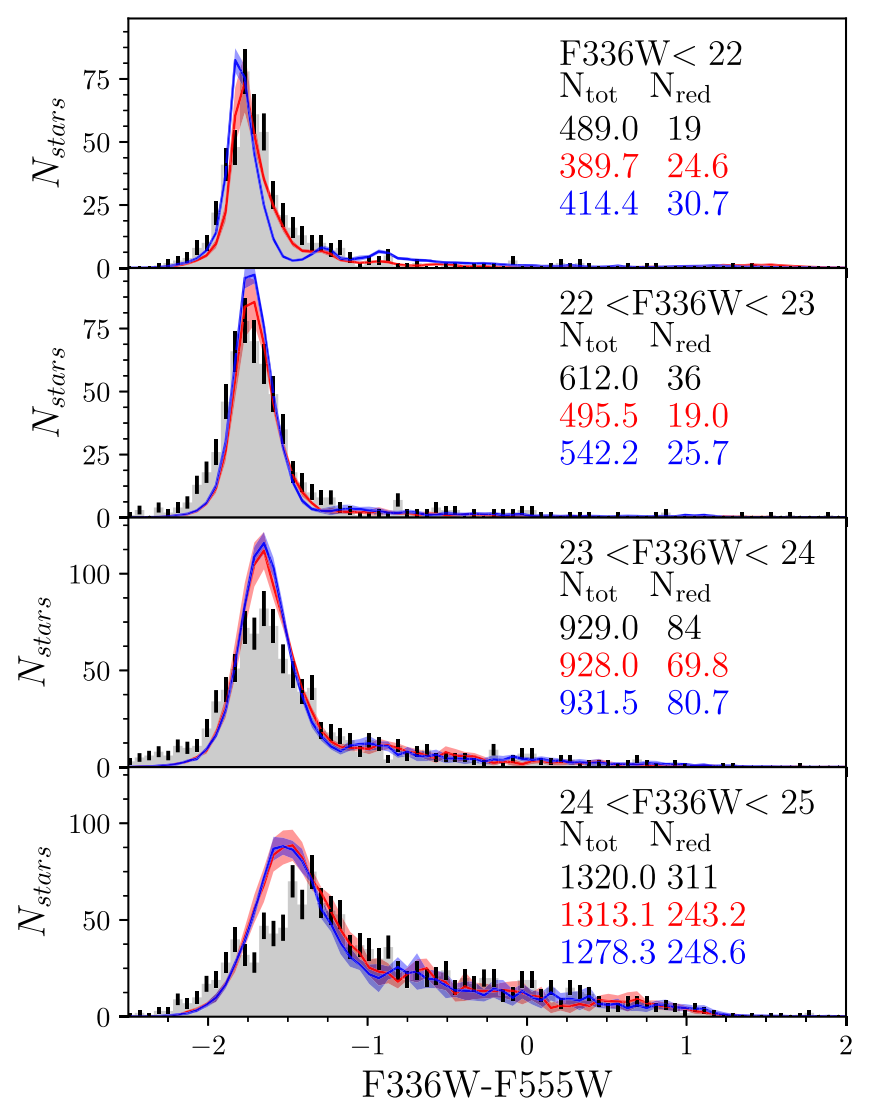

Figure 15. Color distribution of NGC 1705 compared to the models. The symbols are the same as in Figure 8.

the galaxy. From a kinematical point of view, the gas in the central $4 \mathrm{kpc}$ diameter, which corresponds to the brighter part of the optical galaxy, is seen to exhibit rotation in the direction opposite to that of the ellipse and streamers. Moreover, Hunter et al. (1999) found that the regions with the highest velocity dispersions in $\mathrm{HI}$ are located outside the regions of SF and in some cases beyond the bright part of the optical galaxy.

All of these characteristics are consistent with a picture in which NGC 4449 has been disturbed by an external perturber, and the gas has not yet returned to equilibrium. However, in contrast to the disturbed morphology of the gas, NGC 4449 is a fairly isolated system. Its closer neighbor, DDO 125, located at an apparent separation of $41 \mathrm{kpc}$ from the center of NGC 4449 , does not show signs of damage, and its global gas and optical properties are typical of irregular galaxies (Hunter 1997). Between NGC 4449 and DDO 125 no clear bridge has been detected. As discussed in Hunter et al. (1998), with a H I mass of only $10^{8} M_{\odot}$, it should have lost $90 \%$ of its $\mathrm{H}$ I mass in order to account for all of the mass in the streamers. On the other hand, Martínez-Delgado et al. (2012) and Rich et al. (2012) detected and analyzed a stellar tidal stream in the halo of NGC 4449, arguing that this object is the result of the ongoing disruption of a dSph galaxy, while Annibali et al. (2012) found a globular cluster of NGC 4449 that appears associated with two tails of blue stars, possibly the nucleus of a former gas-rich satellite galaxy undergoing tidal disruption by NGC 4449. Finally, using metallicity measurements of the stream, Toloba et al. (2016) suggested a progenitor as massive as Fornax or Sagittarius. Interestingly, an interaction like that could have had a substantial impact on NGC 4449.

\section{3. $N G C 1705$}

NGC 1705 is the most isolated of the three galaxies, since its closest neighbor is at more than $500 \mathrm{kpc}$ (LSBG F157-089). Despite this, NGC 1705 has a warped H I disk (Meurer et al. 1998) that is significantly offset with respect to the stellar component, a possible signature that the outer $\mathrm{H}$ I gas is still in the process of settling down.

Figure 13 shows the recovered SFH for NGC 1705. Overall, the activity of NGC 1705 has been slightly declining for the past 100 Myr. This behavior changed drastically $10 \mathrm{Myr}$ ago, when the SFR increased by a factor of two or more over the 100 Myr averaged SFR. After the peak, the SFR remained constant and no drop is detected. The resulting extinction AV is $0.25 \mathrm{mag}$, while the differential one $d \mathrm{AV}$ is $0.12 \mathrm{mag}$.

To visualize the quality of the reconstruction, Figure 14 shows the best synthetic CMD (middle and right-hand panels) compared to the observational counterpart, while Figure 15 shows the corresponding color distributions. We were unable to perfectly fit the upper end of the CMD, which is likely populated by stars more massive than $40 M_{\odot}$ (see Figure 2 ) and appears to be broader than predicted by the synthetic CMDs. However, as for Ho II, no modification of the assumed IMF exponent, -2.3 , is necessary. The synthetic CMDs show a significant gap between the MS and the post-MS phase that 


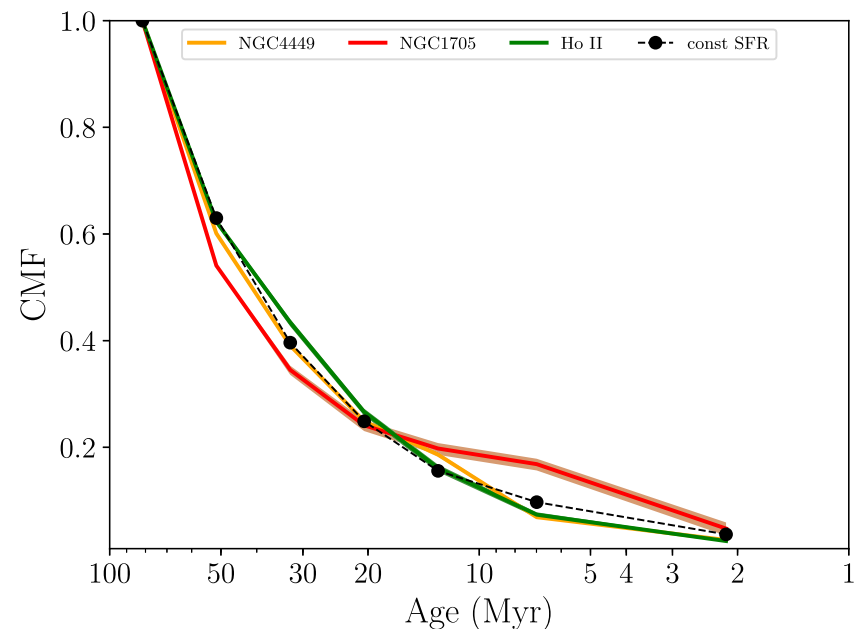

Figure 16. Cumulative stellar mass fraction as a function of time for the three dwarfs and for a constant SFR

instead is completely absent in the observed CMD. Interestingly, the gap holds regardless of the rotational status of the stellar models (PARSEC-COLIBRI models are static and MIST models are rotating).

This discrepancy is not surprising given the uncertainties in the input physics of this mass range (see, e.g., de Mink et al. 2012). Significant mass loss may cause a star to lose over half its mass during its lifetime. This mass loss occurs via stellar winds and strongly affects the stellar evolution of a star. Moreover, massive stars are often found to be rapid rotators and have a significant preference to be in binaries with orbital periods of less than a few days (Sana \& Evans 2011).

Compared to the peak activity of NGC 4449 and Ho II, the peak activity of NGC 1705 is delayed by about $10 \mathrm{Myr}$. Moreover, whereas in NGC 4449 and Ho II the SFR dropped after the peak, the activity of NGC 1705 stayed constant up to now. Our solution is qualitatively similar to the findings of Meurer et al. (1992), Annibali et al. (2003), and Martins et al. (2012), although differences are found in the details.

Meurer et al. (1992) identifies three different populations: an unresolved nucleus, whose activity is consistent with a 5-16 Myr old SF burst; an inner high surface-brightness population, extending out to a radius of $500 \mathrm{pc}$ and characterized by a mean age of $50 \mathrm{Myr}$ with a young component younger than $5 \mathrm{Myr}$ (identified thanks to the presence of Wolf-Rayet emission lines); and a low surfacebrightness population at least $1.5 \mathrm{kpc}$ wide, older than the previous two.

Meurer et al. (1992) studied the $\mathrm{H} \alpha$ emission line kinematics of NGC 1705, detecting the presence of a kiloparsec-scale expanding super-shell of ionized gas centered on the central nucleus with a blueshifted emission component at $540 \mathrm{~km} \mathrm{~s}^{-1}$. Using an expansion model, the authors find an expansion timescale of the order of the age of the nucleus. This evidence, together with the nucleocentric flow geometry, led the authors to conclude that the nucleus itself is the likely power source for the outflow. UV absorption line kinematics bears witness to this, too (Heckman \& Leitherer 1997; Heckman et al. 2001).

Using HST/WFPC2 optical data, Annibali et al. (2003) studied the SFH with the synthetic CMD approach. The authors identified two distinct bursts: an older one (B1) that is confined to the most central regions and occurred from 15 to $10 \mathrm{Myr}$ ago and a stronger young burst (B2) that started $\approx 3$ Myr ago and

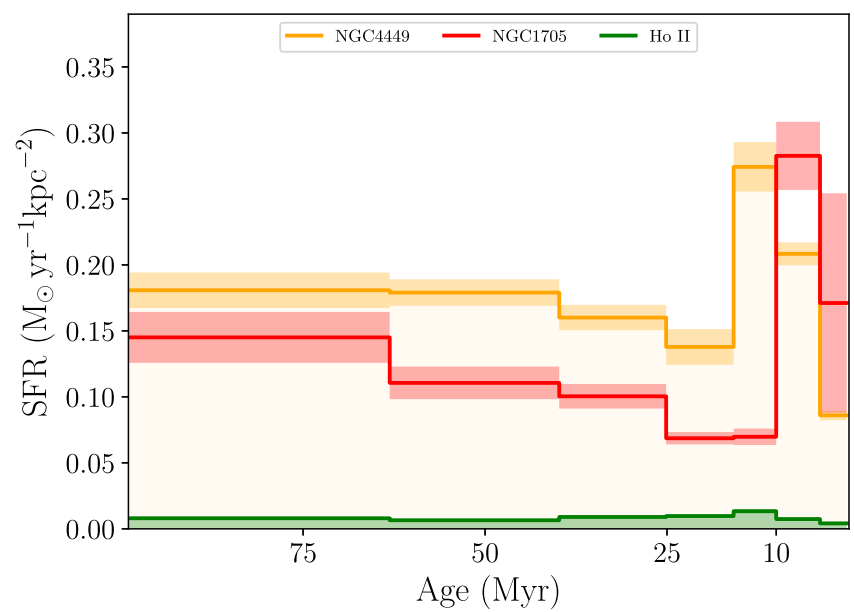

Figure 17. SFR density per unit area for NGC 4449 (gold line), NGC 1705 (red line), and Ho II (green line). For each galaxy, the adopted area is that of the region containing $90 \%$ of the stars in our catalog. Only PARSEC-COLIBRI solutions are shown.

still ongoing with a total SFR of $\approx 0.3 M_{\odot} \mathrm{yr}^{-1}$ (Annibali et al. 2003 adopt a Salpeter IMF between $0.1 M_{\odot}$ and $120 M_{\odot}$ ). More recently, Annibali et al. (2009) restudied the young populations of NGC 1705 at shorter wavelengths (HST/ WFPC2 in the F380W and F439W bands, and HST/ACS/ HRC in the F330W, F555W, and F814W broadband filters), confirming the presence of the younger burst B2. The $50 \mathrm{Myr}$ averaged SFR of Annibali et al. (2003) is shown in Figure 13 with a thick orange dashed line.

Compared to Annibali's et al. solution, our SFH does not show any interruption over the past $10 \mathrm{Myr}$, and our main SF episode starts at an epoch intermediate between those of their two latest SF peaks, but the average rate is in good agreement. A possible origin for the different SFH shapes could be the adopted stellar models: Annibali et al. used the old Padova 1994 models that were available for a few metallicities only, whereas our code incorporates the latest PARSEC-COLIBRI and MIST calculations for a fine grid of metallicities. Moreover, our WFC3 observations have better resolution than the WFPC2 ones, and alleviate the problem of photometric blends.

Compared to integrated emission measurements, our 100 Myr averaged SFR is about a factor of three lower than the rate derived by Lee et al. (2009) using FUV emission (thick green dashed line in Figure 13), while our 10 Myr averaged SFR is only slightly lower than the rate derived by the same authors using $\mathrm{H} \alpha$ nebular emission (thick cyan dashed line in Figure 13). However, these values are not resized to the WFC3 FoV, hence the excess of the FUV SFR may just indicate that the SF activity in the last $100 \mathrm{Myr}$ is more widespread, extending well beyond the WFC3 FoV. Indeed, the much more spatially concentrated distribution of recent and ongoing SF shows only a minor difference with the $\mathrm{H} \alpha$ rate. Moreover, part of the FUV and $\mathrm{H} \alpha$ emission could arise from unresolved clusters and associations, which are not considered in our rates.

Finally, our peak SF is in agreement with that of Martins et al. (2012). This confirms previous studies indicating that the core of NGC 1705 contains massive stars, but no $\mathrm{O}$ and/or Wolf-Rayet objects. Using different evolutionary tracks, they estimate the age of the SSC to be $12 \pm 6 \mathrm{Myr}$. 


\section{Discussion}

A common feature in all recovered SFHs is the existence of a non-zero (at $1 \sigma$ level) SF activity at all ages. While the SFHs present galaxy-to-galaxy variations, particularly at the youngest epochs, the general trend is a relatively flat SF as a function of time. It is important to point out, however, that the time resolution at older ages gets progressively worse, hence SF enhancement similar to those detected in the last $30 \mathrm{Myr}$ could be indistinguishable from a constant activity 100-200 Myr ago.

All three dwarfs show SF enhancements in the last $50 \mathrm{Myr}$, but SF is not always continuing to the present time at a very high rate. The strongest enhancement is found in NGC 4449, which is followed by a lower activity in the last $10 \mathrm{Myr}$. The situation is opposite in NGC 1705, where the highest rate in the last $50 \mathrm{Myr}$ is reached in the last $10 \mathrm{Myr}$. Ho II is qualitatively similar to NGC 4449, even though the ratio between the peak activity and the subsequent drop is much lower. These differences are promptly evident when one compares the cumulative stellar mass fraction (CMF) for the three dwarfs (Figure 16) in the last $100 \mathrm{Myr}$ (NGC 1705's solution becomes too uncertain at older epochs). The CMF, i.e., the fraction of the total stellar mass formed at a given time, provides a normalized measure of the stellar mass accumulation. Compared to the absolute SFHs, cumulative ones are less affected by correlated SFRs in adjacent time bins. In order to combine the PARSEC-COLIBRI and MIST solutions, the CMFs of Figure 16 are the weighted mean ${ }^{27}$ of the two for each galaxy.

The net result is that NGC 1705 systematically deviates from NGC 4449 and Ho II. Using constant mass production (dashed line) as a reference model, while NGC 1705's production is clearly above the reference model in the last $10 \mathrm{Myr}$ (and below the reference model between 30 and 50 Myr), NGC 4449 shows a relative excess of mass between 10 and $16 \mathrm{Myr}$ ago. On the other hand, Ho II is almost indistinguishable from constant mass production.

At this point, an interesting question is whether or not the most recent peak is a unique event in the recent history of our galaxies. Simulations taking into account feedback processes illustrate that $\mathrm{SF}$ in isolated dwarf galaxies can exhibit episodic or cyclic behavior (e.g., Stinson et al. 2007). Here, the starburst is terminated by the expulsion of gas through supernova winds. If the gas later accretes back to the galaxy, a new starburst could be ignited. Concerning our sample, the answer depends on the lookback time. In the last $16 \mathrm{Myr}, \mathrm{NGC} 4449$ assembled a stellar mass between $7.7 \times 10^{6} M_{\odot}$ and $9.0 \times 10^{6} M_{\odot}$. If we dilute this mass between 25 and $40 \mathrm{Myr}$ ago, the resulting SFR (0.52-0.60 $\left.M_{\odot} \mathrm{yr}^{-1}\right)$ would be higher than the measured rate $(0.40-0.46$ $M_{\odot} \mathrm{yr}^{-1}$ ), whereas between 40 and $63 \mathrm{Myr}$ ago the resulting SFR would be lower than the measured rate (hence we cannot exclude multiple events like the current one or stronger). Taking these numbers at face value and considering the error bars of our $\mathrm{SFH}$, we find it unlikely that another SF enhancement similar to the most recent one has taken place in NGC 4449 in the past $40 \mathrm{Myr}$, whereas at older epochs the situation is much more uncertain. In other words, the recent enhancement of NGC 4449 is probably a unique event in the last $40 \mathrm{Myr}$, but nothing can be said for the earlier epochs. From this point of view, an SFH composed of periodic bursts with duration $5 \mathrm{Myr}$, period

\footnotetext{
27 If we consider the two solutions as two normal independent variables with means $\mathrm{z} 1$ and $\mathrm{z} 2$, and dispersions $\sigma_{1}$ and $\sigma_{2}$, the combined solution is normal with mean $\left(\sigma_{1}^{-2} * z 1+\sigma_{2}^{-2} * z 2\right) /\left(\sigma_{1}^{-2}+\sigma_{2}^{-2}\right)$ and dispersion $\left(\sigma_{1}^{-2}+\sigma_{2}^{-2}\right)^{-1 / 2}$.
}

$40 \mathrm{Myr}$, and peak rate like the current one would be indistinguishable from constant activity prior to $40 \mathrm{Myr}$ ago. From a dynamical point of view, using $N$-body simulations, Theis (1999) found that the closest approach between NGC 4449 and the nearby dwarf DDO 125 happened $350 \mathrm{Myr}$ ago at a minimum distance of $25 \mathrm{kpc}$, while Rich et al. (2012) found a timescale of the order of $10^{8} \mathrm{yr}$ for the pericentric passage of NGC 4449B, a dwarf galaxy companion of NGC 4449 in a transient stage of tidal disruption.

In a forthcoming paper, Sacchi et al. (2018), we will explore these older epochs using deeper HST/ACS optical images.

A similar conclusion can be reached for NGC 1705. In the last $10 \mathrm{Myr}$, this galaxy assembled about $5.7 \times 10^{5} M_{\odot}$ of stars. If a similar stellar mass was diluted between 25 and $40 \mathrm{Myr}$ ago, the resulting SFR would be higher than the measured rate, whereas at older epochs it would be comparable (hence suggesting that at these epochs another SF event similar to the current one cannot be ruled out).

Concerning Ho II, the combination of large statistical uncertainties and lower peak rate prevents any conclusion about the presence of previous peaks similar to the current one.

Finally, we must consider the effect of size, since NGC 1705 is significantly more compact than NGC 4449 and Ho II. We thus compare the SFRs per unit area. Since our photometry does not cover the entire extent of the galaxies, we consider the SFR density only over a meaningful portion of our dwarfs, and we have chosen the region in each galaxy whose isodensity contour encloses $90 \%$ of all stars in our catalog. The corresponding area is used to normalize the SFHs, as shown in Figure 17. Of course, this is a crude estimate since the photometric completeness is not the same and different generations do not have the same spatial distribution. In general, the more concentrated the stars are within the galaxy, the more underestimated the star formation density. Interestingly, despite this bias (which disfavors NGC 1705), Figure 17 clearly shows that the projected star formation density peak of NGC 1705 is comparable with that of NGC 4449, and is one order of magnitude higher than that of Ho II.

\section{Conclusions}

The main goal of this paper was a detailed analysis of the UV CMDs of the younger resolved stars in three well-studied active dwarf galaxies, NGC 4449, NGC 1705, and Ho II, not only in terms of quantitative SFHs but also in terms of reliability of the most popular stellar evolution models for intermediate- and high-mass stars. For this task, we have compared deep HST F336W versus F336W - F555W CMDs with synthetic ones generated with state-of-the-art stellar models, namely PARSEC-COLIBRI and MIST. This has allowed us to study the recent history of these galaxies with unprecedented resolution, emphasizing the strengths and weaknesses of these new models. Our main results are summarized as follows.

1. In general, we found good overall agreement between the SFHs derived using PARSEC-COLIBRI and MIST. However, significant systematic deviations emerged as well, suggesting that the physical assumptions of microand macrophysics still have an impact on the recovered rates. Indeed, this result is not surprising given the clear morphological differences already visible in the synthetic CMDs of Figure 5, with both colors and luminosities 
affected. This result is especially important if one considers that PARSEC-COLIBRI and MIST only represent two possible realistic parameterizations, whereas a full estimate of the real uncertainties should take into account different assumptions about critical parameters like stellar mass loss, overshooting, rotation, and binary evolution. From this point of view, the differences between PARSEC-COLIBRI and MIST SFHs should be taken as lower limits to the real uncertainties due to stellar evolution modeling.

Concerning the SFHs, no strong SF burst was detected in the last $180 \mathrm{Myr}$ in any of the three starburst dwarfs. The SFR increases, at most, by a factor of $\approx 2$ over the $100 \mathrm{Myr}$ averaged SFR. This "flickering" SF mode is typically seen in the last $100 \mathrm{Myr}$ of many highresolution studies (see, e.g., Weisz et al. 2008; McQuinn et al. 2010) and is probably connected with the formation of star clusters and associations. Multiple flickering events could then form the long-lasting (>450 Myr) bursts detected by McQuinn et al. (2010) in several dwarfs, whose origin is probably global. Compared with other independent SFR tracers, our 100 Myr averaged SFR and $10 \mathrm{Myr}$ averaged SFR are also in good agreement with FUV and $\mathrm{H} \alpha$ rates, respectively.

Our three dwarfs show qualitatively similar histories: a recent major SF enhancement superimposed on a rather constant SF activity. Quantitatively, instead, the SFRs differ from each other by orders of magnitude, and the details of the most recent peak are remarkably different. The recent SFR in Ho II and NGC 4449 peaked 10-15 Myr ago and has been declining since then, whereas the SF activity in NGC 1705 peaked $10 \mathrm{Myr}$ ago and is still ongoing. Moreover, once normalized to the area, NGC 1705's activity is comparable to that of the far more massive NGC 4449.

Concerning the timing of the most recent peak, the SF enhancements of NGC 4449 and NGC 1705 are probably the only major SF event in the history of these galaxies over the last $40 \mathrm{Myr}$. A similar conclusion cannot be drawn for Ho II, since the peak activity is too low to be detected even $40 \mathrm{Myr}$ ago with current data.

Finally, the truly isolated nature of NGC 1705 compared to NGC 4449 and Ho II suggests that close interactions with neighboring galaxies may not be the only explanation for the recent SF enhancement. Indeed, the clearly disturbed gas kinematics (see, e.g., Lelli et al. 2014) of NGC 1705 could point to a cold accretion of gas as the responsible process for the current SF event.

2. In terms of IMF, a Salpeter-like power law down to $5 M_{\odot}$ is consistent with the data of Ho II and NGC 1705, whereas in NGC 4449 a flatter IMF $(s=-2.0)$ provides a better fit to the data. A similar result has been recently found for the starburst region of 30 Doradus (Schneider et al. 2018). Using spectroscopy, these authors found $32 \% \pm 12 \%$ more stars above $30 M_{\odot}$ than predicted by the standard Salpeter IMF. Interestingly, in NGC 4449, the excess of stars (depending on the model, between $15 \%$ and $23 \%$ with respect to Salpeter) is for magnitudes brighter than $\mathrm{F} 336 \mathrm{~W} \approx 23$, where at least $50 \%$ of the stars are more massive than $20 M_{\odot}$.

3. While all models generally reproduce the expected numbers of MS and post-MS stars in different magnitude bins, the color distributions show several issues that cannot be readily fixed by changing metallicity. The transition from MS to post-MS is especially problematic in NGC 4449, the only one with a statistically well-populated CMD. At the bright end, the total number of massive stars can be reproduced using a flatter IMF, but not the ratio between MS and post-MS stars, with a clear excess of the latter. This behavior is further complicated at the faint end of NGC 4449 (F336W > 23), where all models predict a gap between the MS and post-MS around F336W $\mathrm{F} 555 \mathrm{~W} \approx-1$, whereas the observational $\mathrm{CMD}$ shows a smooth transition. We also found that NGC 4449 is affected by significant differential reddening.

The best synthetic models for Ho II do not show significant discrepancies with the observational counterpart. Interestingly, this galaxy has negligible differential reddening and lower metallicity compared to NGC 4449 , suggesting that both of these factors may have a role in the mismatches of NGC 4449. On the metallicity side, this may suggest a difficulty for the models in matching the BL length at higher metallicity. In this direction, the bestfitting CMDs of the similarly metal-rich NGC 1705 show analogous issues. The most massive stars of NGC 1705 make a smooth transition from the MS to post-MS, with no clear distinction between the two species, whereas all models predict a net gap.

We wish to emphasize once again that the above results have been obtained thanks to the exquisite color and time resolution allowed by UV photometry. Although optical CMDs have the advantage of reaching much older lookback times, thus allowing the SFH to be derived back to much earlier epochs, UV CMDs allow us to separate much better MS and post-MS stars, thus distinguishing in more detail the most recent history. To these two complementary viewpoints, in the future it will be crucial to add also the NIR one, when JWST will allow the reddest evolutionary phases, from the youngest ones of stars still embedded in their parent cocoon to the latest AGB and thermally pulsating stars, to the old red clump ones, to be studied in detail.

We warmly thank Kristy McQuinn for helpful discussions and suggestions. M.C. and M.T. acknowledge funding from the INAF PRIN-SKA 2017 program 1.05.01.88.04. D.A.G. kindly acknowledges financial support by the German Research Foundation (DFG) through programme GO 1659/3-2. B.G.E. acknowledges support from NASA grant HST-GO-13364.14-A.

\section{ORCID iDs}

M. Cignoni (i) https://orcid.org/0000-0001-6291-6813

E. Sacchi (iD https://orcid.org/0000-0001-5618-0109

A. Aloisi (iD https://orcid.org/0000-0003-4137-882X

M. Tosi (ib https://orcid.org/0000-0002-0986-4759

D. Calzetti if https://orcid.org/0000-0002-5189-8004

J. C. Lee (iD https://orcid.org/0000-0002-2278-9407

E. Sabbi (iD https://orcid.org/0000-0003-2954-7643

A. Adamo (i) https://orcid.org/0000-0002-8192-8091

D. O. Cook (iD https://orcid.org/0000-0002-6877-7655

D. A. Dale (i) https://orcid.org/0000-0002-5782-9093

B. G. Elmegreen (iD https://orcid.org/0000-0002-1723-6330

D. A. Gouliermis (1D https://orcid.org/0000-0002-2763-0075

K. Grasha (iD https://orcid.org/0000-0002-3247-5321

E. K. Grebel (iD https://orcid.org/0000-0002-1891-3794 
D. A. Hunter (1) https://orcid.org/0000-0002-3322-9798

K. E. Johnson (1) https://orcid.org/0000-0001-8348-2671

L. J. Smith (1) https://orcid.org/0000-0002-0806-168X

D. A. Thilker (1) https://orcid.org/0000-0002-8528-7340

\section{References}

Annibali, F., Aloisi, A., Mack, J., et al. 2008, AJ, 135, 1900

Annibali, F., Greggio, L., Tosi, M., Aloisi, A., \& Leitherer, C. 2003, AJ, 126,2752

Annibali, F., Nipoti, C., Ciotti, L., et al. 2016, ApJL, 826, L27

Annibali, F., Tosi, M., Aloisi, A., van der Marel, R. P., \& Martinez-Delgado, D. 2012, ApJL, 745, L1

Annibali, F., Tosi, M., Monelli, M., et al. 2009, AJ, 138, 169

Annibali, F., Tosi, M., Pasquali, A., et al. 2015, AJ, 150, 143

Bekki, K., \& Couch, W. J. 2003, ApJL, 596, L13

Bekki, K., \& Freeman, K. C. 2002, ApJL, 574, L21

Bellazzini, M., Ferraro, F. R., \& Pancino, E. 2001, ApJ, 556, 635

Berg, D. A., Skillman, E. D., Marble, A. R., et al. 2012, ApJ, 754, 98

Bernard, E. J., Ferguson, A. M. N., Barker, M. K., et al. 2012, MNRAS, 426, 3490

Bertelli, G., Bressan, A., Chiosi, C., Fagotto, F., \& Nasi, E. 1994, A\&AS, 106, 275

Bressan, A., Girardi, L., Marigo, P., Rosenfield, P., \& Tang, J. 2015, in Asteroseismology of Stellar Populations in the Milky Way, Astrophysics and Space Science Proceedings, Vol. 39 (Cham: Springer), 25

Bressan, A., Marigo, P., Girardi, L., et al. 2012, MNRAS, 427, 127

Bureau, M., \& Carignan, C. 2002, AJ, 123, 1316

Calzetti, D., Lee, J. C., Sabbi, E., et al. 2015, AJ, 149, 51

Cardelli, J. A., Clayton, G. C., \& Mathis, J. S. 1989, ApJ, 345, 245

Choi, J., Dotter, A., Conroy, C., et al. 2016, ApJ, 823, 102

Cignoni, M., Sabbi, E., van der Marel, R. P., et al. 2015, ApJ, 811, 76

Cignoni, M., Sabbi, E., van der Marel, R. P., et al. 2016, ApJ, 833, 154

Croxall, K. V., van Zee, L., Lee, H., et al. 2009, ApJ, 705, 723

Dalcanton, J. J., Williams, B. F., Melbourne, J. L., et al. 2012, ApJS, 198, 6

de Mink, S. E., Brott, I., Cantiello, M., et al. 2012, in ASP Conf. Ser 465, Proceedings of a Scientific Meeting in Honor of Anthony F. J. Moffat (San Francisco, CA: ASP), 65

Dohm-Palmer, R. C., Skillman, E. D., Saha, A., et al. 1997, AJ, 114, 2527

Dolphin, A. E. 2000, PASP, 112, 1383

Dotter, A. 2016, ApJS, 222, 8

Elmegreen, B. G., Zhang, H.-X., \& Hunter, D. A. 2012, ApJ, 747, 105

Evstigneeva, E. A., Drinkwater, M. J., Jurek, R., et al. 2007, MNRAS, 378, 1036

Firth, P., Evstigneeva, E. A., Jones, J. B., et al. 2006, MNRAS, 372, 1856

Gallart, C., Monelli, M., Mayer, L., et al. 2015, ApJL, 811, L18

Gerola, H., Seiden, P. E., \& Schulman, L. S. 1980, ApJ, 242, 517

Girardi, L., Bressan, A., Bertelli, G., \& Chiosi, C. 2000, A\&AS, 141, 371

Harris, J., Zaritsky, D., \& Thompson, I. 1997, AJ, 114, 1933

Heckman, T. M., \& Leitherer, C. 1997, AJ, 114, 69

Heckman, T. M., Sembach, K. R., Meurer, G. R., et al. 2001, ApJ, 554, 1021

Hill, R. S., Home, A. T., Smith, A. M., et al. 1994, ApJ, 430, 568

Huchtmeier, W. K., \& Richter, O. G. 1989, Sci, 246, 943

Hunter, D. 1997, PASP, 109, 937

Hunter, D. A., \& Elmegreen, B. G. 2004, AJ, 128, 2170

Hunter, D. A., van Woerden, H., \& Gallagher, J. S. 1999, AJ, 118, 2184

Hunter, D. A., Wilcots, E. M., van Woerden, H., Gallagher, J. S., \& Kohle, S. 1998, ApJL, 495, L47

Karczewski, O. Ł., Barlow, M. J., Page, M. J., et al. 2013, MNRAS, 431, 2493

Kennicutt, R. C., Jr. 1998, ARA\&A, 36, 189

Kereš, D., Katz, N., Weinberg, D. H., \& Davé, R. 2005, MNRAS, 363, 2

Koleva, M., De Rijcke, S., Zeilinger, W. W., et al. 2014, MNRAS, 441, 452

Kroupa, P. 2001, MNRAS, 322, 231

Lee, J. C., Gil de Paz, A., Tremonti, C., et al. 2009, ApJ, 706, 599
Lelli, F., Verheijen, M., \& Fraternali, F. 2014, MNRAS, 445, 1694

Lelli, F., Verheijen, M., Fraternali, F., \& Sancisi, R. 2012, A\&A, 544, A145

Marigo, P., \& Girardi, L. 2007, A\&A, 469, 239

Marigo, P., Girardi, L., Bressan, A., et al. 2017, ApJ, 835, 77

Martínez-Delgado, D., Romanowsky, A. J., Gabany, R. J., et al. 2012, ApJL, 748, L24

Martins, F., Förster Schreiber, N. M., Eisenhauer, F., \& Lutz, D. 2012, A\&A, 547, A17

McQuinn, K. B. W., Skillman, E. D., Cannon, J. M., et al. 2010, ApJ, 724, 49

McQuinn, K. B. W., Skillman, E. D., Dalcanton, J. J., et al. 2011, ApJ, 740, 48

McQuinn, K. B. W., Skillman, E. D., Dalcanton, J. J., et al. 2012, ApJ, 759, 77

McQuinn, K. B. W., Skillman, E. D., Dolphin, A. E., \& Mitchell, N. P. 2015, ApJ, 808, 109

Melisse, J. P. M., \& Israel, F. P. 1994, A\&A, 285, 51

Meurer, G. R., Freeman, K. C., Dopita, M. A., \& Cacciari, C. 1992, AJ, 103,60

Meurer, G. R., Staveley-Smith, L., \& Killeen, N. E. B. 1998, MNRAS, 300,705

Meynet, G., \& Maeder, A. 1997, A\&A, 321, 465

Moustakas, J., Kennicutt, R. C., Jr., Tremonti, C. A., et al. 2010, ApJS, 190, 233

Patton, D. R., Torrey, P., Ellison, S. L., Mendel, J. T., \& Scudder, J. M. 2013, MNRAS, 433, L59

Paxton, B., Bildsten, L., Dotter, A., et al. 2011, ApJS, 192, 3

Paxton, B., Cantiello, M., Arras, P., et al. 2013, ApJS, 208, 4

Paxton, B., Marchant, P., Schwab, J., et al. 2015, ApJS, 220, 15

Puche, D., Westpfahl, D., Brinks, E., \& Roy, J.-R. 1992, AJ, 103, 1841

Ramya, S., Kantharia, N. G., \& Prabhu, T. P. 2009, in ASP Conf. Ser. 407, The Low-Frequency Radio Universe, ed. D. J. Saikia et al. (San Francisco, CA: ASP), 114

Rich, R. M., Collins, M. L. M., Black, C. M., et al. 2012, Natur, 482, 192

Sabbi, E., Calzetti, D., Ubeda, L., et al. 2018, ApJS, in press (arXiv:1801. 05467)

Sabbi, E., Lennon, D. J., Anderson, J., et al. 2016, ApJS, 222, 11

Sabbi, E., Lennon, D. J., Gieles, M., et al. 2012, ApJL, 754, LL37

Sacchi, E., Annibali, F., Cignoni, M., et al. 2016, ApJ, 830, 3

Sacchi, E., Cignoni, M., Aloisi, A., et al. 2018, ApJ, submitted

Sana, H., de Koter, A., de Mink, S. E., et al. 2013, A\&A, 550, A107

Sana, H., \& Evans, C. J. 2011, in IAU Symp. 272, Active OB Stars: Structure, Evolution, Mass Loss, and Critical Limits (Cambridge: Cambridge Univ. Press), 474

Schneider, F. R. N., Izzard, R. G., Langer, N., \& de Mink, S. E. 2015, ApJ, 805,20

Schneider, F. R. N., Sana, H., Evans, C. J., et al. 2018, Sci, 359, 69

Starkenburg, T. K., Helmi, A., \& Sales, L. V. 2016, A\&A, 595, A56

Stewart, S. G., Fanelli, M. N., Byrd, G. G., et al. 2000, ApJ, 529, 201

Stierwalt, S., Besla, G., Patton, D., et al. 2015, ApJ, 805, 2

Stierwalt, S., Liss, S. E., Johnson, K. E., et al. 2017, NatAs, in press (arXiv:1701.01731)

Stinson, G. S., Dalcanton, J. J., Quinn, T., Kaufmann, T., \& Wadsley, J. 2007, ApJ, 667, 170

Tang, J., Bressan, A., Rosenfield, P., et al. 2014, MNRAS, 445, 4287

Tang, J., Bressan, A., Slemer, A., et al. 2016, MNRAS, 455, 3393

Theis, C. 1999, RvMA, 12, 309

Toloba, E., Guhathakurta, P., Romanowsky, A. J., et al. 2016, ApJ, 824, 35

Tolstoy, E., Hill, V., \& Tosi, M. 2009, ARA\&A, 47, 371

Tosi, M., Sabbi, E., Bellazzini, M., et al. 2001, AJ, 122, 1271

Tully, R. B. 1988, AJ, 96, 73

van Woerden, H., Bosma, A., \& Mebold, U. 1975, in Coll. Int. CNRS 241, La Dynamique des Galaxies Spirales, ed. L. Weliachew (Paris: Editions du CNRS), 483

Weisz, D. R., Dalcanton, J. J., Williams, B. F., et al. 2011, ApJ, 739, 5

Weisz, D. R., Skillman, E. D., Cannon, J. M., et al. 2008, ApJ, 689, 160

Weisz, D. R., Skillman, E. D., Cannon, J. M., et al. 2009, ApJ, 704, 1538 